\title{
Simulation of Solid Oxide Iron-Air Battery: Effects of Heat and Mass Transfer on Charge/Discharge Characteristics
}

\author{
Hiroko Ohmori $^{\text {a, b, } * \text {, Hiroshi Iwai }}{ }^{\text {a }}$ \\ ${ }^{a}$ Department of Aeronautics and Astronautics, Kyoto University, \\ Nishikyo-ku, Kyoto 615-8540, Japan \\ ${ }^{\mathrm{b}}$ Corporate R\&D Headquarters, Konica Minolta, Inc., \\ Takatsuki City, Osaka 569-8503, Japan
}

*Corresponding author. Tel.: +81-72-6856159; Fax: +81-72-6856114

E-mail address: hiroko.ohmori@konicaminolta.com 


\begin{abstract}
A time-dependent 2-D numerical simulation was performed on a solid oxide iron-air battery (SOIAB) to reveal the fundamental characteristics of this new system. The SOIAB is a rechargeable battery consisting of a solid oxide electrochemical cell (SOEC) and iron as a redox metal. A simple battery configuration was employed assuming a system with a small capacity. A simulation model for a unit element was developed considering heat and mass transfer in the system, taking both electrochemical and redox reactions into account. The numerical results showed the spatial and temporal changes in the temperature field in the charge and discharge operations, which were due to the combined effects of heat generation/absorption by the electrochemical and redox reactions and heat exchange with the air supplied through convective heat transfer. As the reaction rates are functions of the local temperature, the predicted results show the importance of considering the heat transfer phenomena in this system. It was also found that the active reaction region in the redox metal evolves with time. The nonuniform distribution of iron utilization is affected by the effective gas diffusion coefficients in the porous redox metal, and consequently the change in the current density distribution in the SOEC.
\end{abstract}

\title{
Key words:
}

Rechargeable metal-air battery; Solid oxide electrochemical cell; Redox metal; Heat Transfer; Numerical Simulation 


\section{Introduction}

Energy storage devices with large capacity are urgently required as a buffer against the fluctuating power supply from renewable energy sources. Among the various types of rechargeable batteries, metal-air batteries have attracted considerable attention owing to their large capacity due to the unnecessity of oxidizer storage at the cathode [1-5].

A new-concept rechargeable battery consisting of a solid oxide electrochemical cell (SOEC) and iron as a redox metal, i.e., a solid oxide iron-air battery (SOIAB), is also classified as a metal-air battery [6-17]. In this battery system, the SOEC directly converts electrical energy into chemical energy (hydrogen/steam), which is stored in a redox metal couple $\left(\mathrm{Fe}-\mathrm{FeO}_{\mathrm{x}}\right)$. Compared with usual metal-air batteries, a major advantage of the SOIAB is the physical separation of the SOEC and the redox metal, which is enabled by the insertion of a gaseous hydrogen/steam mixture as an oxygen carrier between the two components. This can lead to cycle stability and easy system integration.

Similarly to solid oxide fuel cells, many different cell designs and system configurations are possible for SOIABs [6-17]. The proposed system configurations reported in the literature can be classified into two groups. In the first group, the SOEC and the redox metal are placed in different containers, and the two containers are connected via pipes to allow recirculation of the hydrogen/steam mixture $[13,17]$. In this configuration, suitable operating temperatures for the SOEC and the redox metal can be separately selected. The system can be slightly complicated because a high-temperature blower is required for hydrogen/steam recirculation. This makes the configuration more suitable for large-scale applications. In the other group, the SOEC and the redox metal are packed in a single package $[10,11,16]$. In the gap between the SOEC fuel electrode and the redox metal, the hydrogen/steam mixture naturally diffuses without any additional power input. That is, a blower for the hydrogen/steam 
mixture is not needed in this configuration and therefore the system is simpler. Because the SOEC and the redox metal are physically close in this configuration, an operating temperature suitable for both electrochemical and redox reactions must be chosen. Considering the heat generation/absorption by the reactions, it can be considered that this configuration is more challenging from the viewpoint of thermal management.

In this study we focus on the latter configuration. We previously presented the results of a time-dependent 1-D simulation of the effects of gas diffusion on an SOIAB with this configuration [16]. As we mainly focused on the distribution of gas species and its effects, we limited the calculation domain to a small part of the battery and assumed a uniform temperature distribution while neglecting the effects of heat transfer in the previous study. More recently, Guo et al. [9] conducted a CFD-based 2-D simulation also considering both electrochemical and redox reactions. The battery geometry was set to reflect their experimental setup and they obtained good agreement between the simulation and experimental results. As the operating temperature of their laboratory-scale experiments was controlled using an electric furnace, it was reasonable that they assumed a uniform temperature in the simulation. In a real system, however, the effects of the distributions of the local temperature and gas concentration will be significant.

Clarification of the temperature and concentration fields in an operating system is important but it is generally difficult to experimentally acquire such data. Simulation of the inside of the battery should provide effective guidelines for improving the performance. The main aim of this study is to reveal the fundamental characteristics of an SOIAB by 2-D numerical simulation focusing on the time-dependent distributions of the local temperature and gas concentration. We also focus on the evolution of the redox metal reaction area. 


\section{Numerical Procedure}

\section{2-1. Outline of Battery}

Figure 1 schematically explains the concept of the battery. The battery consists of two main components, a redox metal and an SOEC. The redox metal is typically in the form of fine particles, and iron is assumed in this study. Because the SOIAB is a new concept battery, there is still no established battery geometry at the moment. We assume the following geometry as a simple geometry. The redox metal is packed at the bottom of a rectangular parallelepiped container. The top of the container is originally open and is capped with a planar SOEC with its fuel electrode facing to the redox metal. Inside of the container is filled with the gaseous mixture of hydrogen and steam. Above the air-side electrode, we assume an adiabatic wall to form an air channel. The air is supplied from the left-side in Fig. 1, flows along the air electrode and exhausted to the right-side.

In the discharge operation shown in Fig.1 (a), the SOEC acts as a fuel cell generating electric power as follows;

$$
\begin{array}{ll}
\text { Air-side electrode: } & 1 / 2 \mathrm{O}_{2}+2 \mathrm{e}^{-} \rightarrow \mathrm{O}^{2-}, \\
\text { Fuel-side electrode: } & \mathrm{H}_{2}+\mathrm{O}_{2^{-}} \rightarrow \mathrm{H}_{2} \mathrm{O}+2 \mathrm{e}^{-} .
\end{array}
$$

The generated steam is transported to the redox metal by gas diffusion. It oxidizes the redox metal into a metal oxide as follows;

$$
\text { The redox metal: } \quad 3 \mathrm{Fe}+4 \mathrm{H}_{2} \mathrm{O} \rightarrow \mathrm{Fe}_{3} \mathrm{O}_{4}+4 \mathrm{H}_{2} \text {. }
$$

The generated hydrogen diffuses to the SOEC, and this cycle is repeated in the system. Note that when iron is oxidized, the iron oxide can be wustite $(\mathrm{FeO})$, magnetite $\left(\mathrm{Fe}_{3} \mathrm{O}_{4}\right)$, or hematite $\left(\mathrm{Fe}_{2} \mathrm{O}_{3}\right)$ depending on the temperature and oxygen partial pressure. According to the potential diagram of the Fe-O system $[8,11,12]$, the dominant redox reaction in this study is between iron and magnetite because we 
assume a relatively low temperature of approximately 520-600 ${ }^{\circ} \mathrm{C}$. The formation of magnetite was also confirmed in a preliminary experiment.

When all (or a certain amount) of the redox metal is oxidized, the battery must be recharged. In the charge operation shown in Fig.1 (b), all the above reactions proceed in the reverse direction. The SOEC acts as an electrolyzer

$$
\begin{array}{ll}
\text { Air-side electrode: } & \mathrm{O}^{2-} \rightarrow 1 / 2 \mathrm{O}_{2}+2 \mathrm{e}^{-} \\
\text {Fuel-side electrode: } & \mathrm{H}_{2} \mathrm{O}+2 \mathrm{e}^{-} \rightarrow \mathrm{H}_{2}+\mathrm{O}^{2-}
\end{array}
$$

and the metal oxide is reduced by hydrogen.

The redox metal: $\quad \mathrm{Fe}_{3} \mathrm{O}_{4}+4 \mathrm{H}_{2} \rightarrow 3 \mathrm{Fe}+4 \mathrm{H}_{2} \mathrm{O}$

When all (or a certain amount) of the redox metal is reduced, the charge operation is completed and the system is ready for the next discharge. From the above reactions, the total reaction in the charge/discharge operation can be written as

$$
3 \mathrm{Fe}+2 \mathrm{O}_{2} \stackrel{\text { discharge }}{\stackrel{\text { charge }}{\longleftarrow}} \mathrm{Fe}_{3} \mathrm{O}_{4} .
$$

Essentially, reaction (7) is similar to that of a typical iron-air battery. A major advantage of the SOIAB, however, is the physical separation of the electrochemical reaction (SOEC) from the redox reaction (the redox metal). This separation prevents structural damage that may occur from the change in volume of the redox metal associated with the redox reaction. It also gives us freedom to independently design the power and capacity of the battery since the system output power depends on the total area of the SOEC electrode while the battery capacity is proportional to the amount of the redox metal.

The direction of the reversible metal redox reaction is governed by the composition of the gaseous hydrogen/steam mixture in the container. If the hydrogen partial pressure is greater than the equilibrium pressure of the redox reaction (eqs. (3) and (6)), the reduction is dominant, and vice versa. The hydrogen partial pressure is determined by hydrogen generation/absorption by the SOEC and the redox metal. As 
metal particles can easily be filled into spaces with various shapes, both tubular and planar SOECs are possible for this battery. Packed fine particles of the redox metal are used to ensure a large surface area. We treat the packed particles as a porous material in this study. When the redox metal is oxidized, its volume increases and the pore space in which the gas species diffuse is reduced. Therefore, a change in the state of the porous material affects the gas diffusion and redox reaction rate. With iron as the redox metal, the theoretical capacity density is $1280 \mathrm{Ah} / \mathrm{kg}-\mathrm{Fe}$, corresponding to the amount of hydrogen generated from iron.

\section{2-2. Battery Design and Computational Domain}

Figure 2 schematically shows the configuration of the battery, which consists of an SOEC, the redox metal, a container, and an air channel. We set the $x$ - and $y$-coordinates in the air flow direction and the SOEC thickness direction, respectively. For simplicity, we assume the system is long in $z$-direction (perpendicular to the $x-y$ plane) and focus only on the center region. That is, we assume uniform distribution of all values in $z$-direction and consider the phenomena are two-dimensional. We further simplify the air flow and the SOEC parts and we modeled each of these parts to be one dimensional in $x$-direction. The models are to be explained in the next section. The computational domain is marked with the red and blue broken line in Fig.2. The space inside the container is filled with the redox metal and the gaseous mixture of hydrogen and steam. As this space is isolated from the surrounding region and no external force is applied, the mass transfer inside the container is dominated by diffusion. The amount of the redox metal per unit area of the SOEC electrode is set at $1,5,10$, or $20 \mathrm{~g} / \mathrm{cm}^{2}$. The porosity of the redox metal is assumed to be 0.37 when it is fully oxidized.

For simplicity, the container wall is assumed to be very thin and that its heat capacity is negligibly small. The container is thermally insulated and, therefore, heat 
input to or heat release from the SOIAB occurs only through the heat exchange with the air flow, which is constantly supplied at a temperature of $550{ }^{\circ} \mathrm{C}$. The depth of the redox metal $\left(L_{2}\right)$ is related to the amount of the redox metal per area in the SOEC electrode. $1 \mathrm{~g} / \mathrm{cm}^{2}$ of reduced redox iron results in $L_{2}$ of $4.2 \mathrm{~mm}$. Other design parameters of the system are as follows, length in $x$-direction, $L_{l}=100 \mathrm{~mm}$, distance between redox metal and SOEC, $L_{3}=10 \mathrm{~mm}$, thickness of the SOEC, $L_{4}=1.0 \mathrm{~mm}$, and height of air channel, $L_{5}=2.0 \mathrm{~mm}$.

\section{2-3. Numerical Modeling}

The computational domain shown in Fig.2 is divided into two 1-D simulations and a 2-D simulation. They are all interrelated through the electrochemical reaction and heat transfer. The heat and mass generation/absorption caused by the reactions are considered as source terms. In 1-D simulations, the effects of heat transfer between the solid phase and gas phase appear as a source term as well.

\section{2-3-1. 1-D Simulation Models for Air Flow and SOEC}

As explained in the previous section, the air flow and the SOEC are treated as 1-D simulations in the $x$-direction, respectively. For the air flow, the mass and energy balances are considered neglecting the pressure drop along the channel.

$$
\begin{array}{ll}
\text { Mass: } & \frac{\partial \rho_{a}}{\partial t}+\frac{\partial\left(\rho_{a} u_{a}\right)}{\partial x}=S_{a, \text { mass }} \\
\text { Energy: } & \frac{\partial\left(\rho_{a} C_{p, a} T_{a}\right)}{\partial t}+\frac{\partial\left(\rho_{a} C_{p, a} u_{a} T_{a}\right)}{\partial x}=S_{a, \text { heat }}
\end{array}
$$

where $\rho_{a}, C_{p, a}$ are mass density and specific heat of the binary mixture gas of nitrogen and oxygen flowing in the air channel. $u_{a}$ is the velocity of the flow. $T_{a}$, and $T_{\mathrm{SOEC}}$ are the local temperature of the flow and the SOEC, respectively. $S_{a \text {, mass }}$ and $S_{a \text {, heat }}$ are source terms. Reflecting the oxygen consumption/generation by the electrochemical

reaction, $S_{a \text {, mass }}$ is calculated as $\mp \frac{i \mathrm{M}_{\mathrm{O}_{2}}}{4 F} \frac{1}{L_{5}}$, where $\mathrm{M}_{\mathrm{O} 2}, F$ and $i$ are the molecular 
weight of oxygen, Faraday constant and local current density. The sign is negative in discharge operation. $S_{a, \text { heat }}$ is associated with the convective heat transfer between the flow and the SOEC. It is expressed as $h\left(T_{\mathrm{SOEC}}-T_{a}\right) / L_{5} . h$ is the heat transfer coefficient evaluated by assuming a constant Nusselt number of 4.86 .

The SOEC is also treated as 1-D simulation in the $x$-direction. The energy equation is solved.

$$
\text { Energy: } \frac{\partial\left(\rho_{\mathrm{SOEC}} C_{p, \mathrm{SOEC}} T_{\mathrm{SOEC}}\right)}{\partial t}=\frac{\partial}{\partial x}\left(\lambda_{\mathrm{SOEC}} \frac{\partial T_{\mathrm{SOEC}}}{\partial x}\right)+S_{\mathrm{SOEC}} \text {, heat }
$$

where $\rho_{\mathrm{SOEC}}, C_{p, \mathrm{SOEC}}, \lambda_{\mathrm{SOEC}}$ are mass density, specific heat and thermal conductivity of the SOEC. Note that averaged values are used for these properties, evaluated considering the materials and thicknesses of the electrodes and electrolyte. The source term, $S_{\mathrm{SOEC}}$, heat can be divided into three terms. The first term corresponds to the convective heat transfer between the air flow and the SOEC, $h\left(T_{a}-T_{\mathrm{SOEC}}\right) / L_{4}$. The second term corresponds to the heat exchange between the binary gas in the container

and the SOEC, $-\lambda_{\text {gas }}\left(\partial T_{\text {gas }} / \partial y\right)_{\text {SOEC surface }} / L_{4} . \lambda_{\text {gas }}$ is the thermal conductivity of the binary gas. The last term is the heat generation/absorption associated with the electrochemical reaction and the overpotentials in the SOEC and it is explained in the section 2-3-4.

\section{2-3-2. 2-D Simulation Model in the Container}

A time-dependent 2-D numerical simulation based on energy and mass diffusion equations is conducted inside the container marked with the red broken line in Fig.2. The 2-D simulation domain is further divided into two regions: the porous redox metal and the space between the SOEC and the redox metal. By choosing appropriate physical properties corresponding to each region, the governing equations can be written in the same form. 


$$
\begin{array}{ll}
\text { Energy equation: } & \frac{\partial\left(\rho C_{p} T\right)}{\partial t}=\frac{\partial}{\partial x}\left(\lambda \frac{\partial T}{\partial x}\right)+\frac{\partial}{\partial y}\left(\lambda \frac{\partial T}{\partial y}\right)+S_{\text {heat }} \\
\text { Mass transfer equation: } & \frac{\partial Y_{j}}{\partial t}=\frac{\partial}{\partial x}\left(D \frac{\partial Y_{j}}{\partial x}\right)+\frac{\partial}{\partial y}\left(D \frac{\partial Y_{j}}{\partial y}\right)+S_{\text {mass }, j}
\end{array}
$$

Where $\rho, C_{p}, T, \lambda, S, Y, j, D$ are mass density, specific heat, temperature, thermal conductivity, sources, concentration of species, species, and gas diffusion coefficient, respectively. Note that convection terms do not appear in these equations. Because of the small characteristic length, $L_{3}$, and the temperature difference, the buoyancy force resulting from the variation in gas density cannot overcome the resistance caused by fluid viscosity, that is, the Rayleigh number is much smaller than its critical value. Natural convection does not occur under the calculation conditions set in this study and the gas transfer is governed by the diffusion process. Heat and mass generation associated with the reactions appear as source terms, $S_{\text {heat }}$ or $S_{\text {mass, } j}$ in the equations. They are explained in the sections 2-3-3 and 2-3-4.

\section{2-3-3. Redox Reaction Model of Metal}

Although the redox reaction of iron involves complex elementary reactions, we simplified it to the overall reactions expressed by eqs. (3) and (6) in this study. The reaction rates were obtained from a preliminary experiment using fine iron particles. Prior to the simulation, we performed thermogravimetry measurement (TG) for the iron particles supplying the mixture gas of hydrogen and steam. The exhaust gas composition was measured by using a mass spectrometry. The reaction rate is expressed in terms of the partial pressures of hydrogen and steam, $p_{\mathrm{H} 2}$ and $p_{\mathrm{H} 2 \mathrm{O}}$ and the molar density of the reactant, $M_{i}$ in this study. The reactant is Fe in discharge operation while it is $\mathrm{Fe}_{3} \mathrm{O}_{4}$ in charge operation. Note that not only $p_{\mathrm{H} 2}$ and $p_{\mathrm{H} 2 \mathrm{O}}$ but also $M_{i}$ is a function of time and location since the reactant, $\mathrm{Fe}$ or $\mathrm{Fe}_{3} \mathrm{O}_{4}$, is consumed during the discharge and charge operations. The reaction rate is used to calculate the 
source terms. For example, the source term of eq. (12) for hydrogen is evaluated using the local values of $p_{H 2}, p_{H 2 O}$ and $M_{i}$ as

Discharge:

$$
S_{\text {mass }, \mathrm{H}_{2}}=\left\{-\left(k_{1} \times p_{\mathrm{H}_{2}}\right)+\left(k_{2} \times p_{\mathrm{H}_{2} \mathrm{O}}\right)\right\} \times M_{F e}
$$

Charge:

$$
S_{\text {mass }, H_{2}}=\left\{-\left(k_{1} \times p_{H_{2}}\right)+\left(k_{2} \times p_{H_{2} O}\right)\right\} \times 3 M_{F e 3 O 4}
$$

where $k_{1}$ and $k_{2}$ are reaction coefficients. The values of reaction coefficients were evaluated by fitting the experimental data obtained by TG measurements. The temperature dependence of the reaction rates is also considered by changing the reaction coefficients in accordance with the temperature. Here, the rate is proportional to only the molar density of the reactant assuming that the activity of the solid phase (Fe or $\mathrm{Fe}_{3} \mathrm{O}_{4}$ ) is unity. When the operations proceed and $\mathrm{Fe}$ or $\mathrm{Fe}_{3} \mathrm{O}_{4}$ is almost consumed up, the reaction rate decreases sharply and the discharge or charge operation comes to an end. The molar density of $\mathrm{Fe}, M_{F e}$, is related to the state of charge, SOC, which is an important indicator of the battery condition. The SOC is explained in the latter section.

The reduced gas diffusion inside the porous metal was taken into account by considering an effective diffusion coefficient, $D_{\text {eff. }}$. Here, we use the simple expression, $D_{\text {eff }}=D_{0} \cdot \varepsilon / \tau$, where $\varepsilon$ and $\tau$ are the porosity and tortuosity factor of the porous material, respectively. $D_{0}$ is the diffusion coefficient in a free space without porous material. The tortuosity factor is assumed to be the reciprocal of the porosity, $\tau=1 / \varepsilon$ [18].

We further assume that the representative pore diameter is much larger than the mean free path of hydrogen and we neglect Knudsen diffusion. The effective thermal conductivity of the porous region is modeled as $\lambda_{\text {eff }}=\varepsilon \lambda_{\text {gas }}+(1-\varepsilon) \lambda_{\text {metal. }}$ As we assume iron to be the redox metal, its volume increases by a factor of 2.1 when it 
is oxidized. The volume expansion/shrinkage associated with the redox reaction is considered via the change in the local porosity of the redox metal. When the redox metal is fully reduced to $\mathrm{Fe}$ the porosity is 0.7 and when it is fully oxidized to $\mathrm{Fe}_{3} \mathrm{O}_{4}$ the porosity is 0.37 . It means that the $D_{\text {eff }}$ discussed above takes a value in a range of $0.14 \leq D_{\text {eff }} / D_{0} \leq 0.49$, depending on the local porosity.

Iron oxidation and reduction are exothermic and endothermic reactions, respectively. The reaction heat is evaluated from the enthalpy change, $\Delta H$, of eqs. (3) and (6), respectively, considering its temperature dependency [19]. The local reaction rate is evaluated from the local $p_{H 2}, p_{H 2 O}, M_{i}$ and temperature and by considering the reaction heat, $\Delta H$, the local heat generation or absorption is obtained and is applied to the energy equation as a source term, $S_{\text {heat }}$.

\section{2-3-4. Reaction Model at SOEC}

There will be nonuniform distributions of the temperature and gas concentrations and, consequently, of the current density along the air-flow direction ( $x$-direction) in the SOEC. In this simulation, the average current density is set at 200 $\mathrm{mA} / \mathrm{cm}^{2}$ and the terminal voltage is assumed to be uniform throughout the area, assuming high electric conductivity of the electrodes. The current is assumed to flow only in the thickness direction in the SOEC. The electromotive force, $E$, is locally evaluated by the Nernst equation,

$$
\begin{aligned}
& E=E^{0}+\frac{R T}{2 F} \ln \left(\frac{p_{\mathrm{H}_{2}} \times p_{\mathrm{O}_{2}}^{1 / 2}}{p_{\mathrm{H}_{2} \mathrm{O}}}\right) \\
& E^{0}=\frac{\Delta G^{0}}{2 F}
\end{aligned}
$$

where $E_{0}, R, F$, and $\Delta G$ are the standard electromotive force, gas constant, Faraday constant and Gibbs free energy change, respectively. The concentration overpotential is neglected. The IR loss, $\eta_{o h m}$, is expressed as $\eta_{o h m}=A S R_{o h m} * i$, where $i$ and $A S R_{o h m}$, 
are the local current density and area-specific resistance, respectively. $A S R_{\text {ohm }}$ was set to be $1 \mathrm{e}-5\left[\Omega \mathrm{m}^{2}\right]$ by fitting to an $i$ - $V$ curve obtained from an experiment performed at $600{ }^{\circ} \mathrm{C}[20]$ and was assumed to be constant. The activation overpotential, $\eta_{\text {act }}$, is evaluated by a Butler-Volmer type equation [21]

$$
i=i_{0}\left[\exp \left(\frac{2 \mathrm{~F} \eta_{a c t}}{\mathrm{RT}}\right)-\exp \left(\frac{-\mathrm{F} \eta_{a c t}}{\mathrm{RT}}\right)\right]
$$

The exchange current density, $i_{0}$, is generally a function of local temperature and gas concentrations. In this study, however, its value is assumed to be constant at a value estimated at $600{ }^{\circ} \mathrm{C}$ [20]. It is still difficult to find conclusive experimental data available that cover both hydrogen oxidation and steam electrolysis using practically same cells under low temperature condition set in this study, $500-650{ }^{\circ} \mathrm{C}$. Then the terminal voltage, $V$, can be obtained as

$$
\begin{array}{ll}
\text { Discharge : } & V_{\text {discharge }}=E-\left(\eta_{\text {ohm }}+\eta_{\text {act }}\right) \\
\text { Charge : } & V_{\text {charge }}=E+\left(\eta_{\text {ohm }}+\eta_{\text {act }}\right)
\end{array}
$$

The generation/consumption of oxygen associated with the electrochemical reaction on the air-electrode is included in the calculation as a source term (see section 2-3-1). The generation/consumption of hydrogen or steam associated with the electrochemical reactions on the fuel-side electrode are also included in the calculation as a boundary condition at the surface of the electrode. It is introduced to the calculation as a source term of the gas-phase grid adjacent to the SOEC electrode. They are evaluated from the local current density as shown in Table 1.

The hydrogen oxidation in discharge operation and the steam electrolysis reaction in charge operation at the SOEC are exothermic and endothermic, respectively. In these reactions, the thermal energy associated with $T \Delta S$ of eqs. (3) or (6) is released or absorbed. $\Delta S$ is the entropy change of the reaction. The local reaction rate is obtained from the local current density and by considering $T \Delta S$, the 
heat generation or absorption is calculated and is included in the source term of the energy equation of the SOEC, $S_{\mathrm{SOEC}}$, heat of eq. (10). In addition to this, there are heat generation caused by inner resistance and activation overpotential during both hydrogen oxidation and steam electrolysis. The heat generation associated with Joule heating, $i^{2} \times A S R_{o h m}$, and activation overpotential, $i \times \eta_{a c t}$, are also included in the source term of the energy equation of the SOEC, $S_{\mathrm{SOEC} \text {, heat }}$ of eq. (10).

\section{2-3-5. Numerical Procedure}

An in-house program code was applied to the battery model shown in Fig.2. The governing equations were discretized using the finite volume method. A nonuniform grid system was applied with finer grid spacing in the porous redox metal medium, where a steep concentration gradient was expected. A fully implicit method was used to solve Eqs. (11) and (12). The time increment was set at $0.2 \mathrm{~s}$ for the iron density of $1 \mathrm{~g} / \mathrm{cm}^{2}$. A systematic test was conducted to confirm that the results were independent of the grid or time increment. The boundary conditions for eqs. (11) and (12) are summarized in Table 1 and Table 2. Note that the effects of the electrochemical and redox reactions are taken into account through the source terms

\section{2-3-6. Computational Conditions.}

We conduct two types of calculations in this study. The first type is conducted for a noncyclical simple discharge/charge operation to determine the fundamental characteristics of the system. The redox metal is completely reduced/oxidized at the beginning of the discharge/charge operation and set as the initial condition. We define the state of charge, SOC, as the ratio of the capacity stored in the battery to that at full charge. It is evaluated based on the molar density of Fe. At an extreme condition when the battery is fully charged, all iron exists as Fe. If we write the molar density of $\mathrm{Fe}$ at this condition as $M_{F e}{ }^{0}$, the local SOC during the 
operation can be expressed as $M_{F e} / M_{F e}{ }^{0}$ using the instantaneous value of $M_{F e}$ at each location. The battery's total SOC can be calculated taking the average of the local SOC. The SOC is $100 \%$ when the battery is fully charged. Because the current density is kept constant in each operation, the elapsed time is proportional to SOC.

After the single discharge/charge calculations, a calculation of the discharge/charge cycle operations is conducted. SOC is set at $100 \%$ as the initial condition. When SOC reaches $50 \%$ in the first discharge operation, the operation mode is switched to the charge operation and the battery is charged until SOC reaches 98\%. The discharge and charge operations are sequentially repeated three times with SOC varied between $50 \%$ and $98 \%$.

In both calculations, the system is initially set at $550{ }^{\circ} \mathrm{C}$ with a total pressure of $1 \mathrm{~atm}$. The SOEC is operated in a constant-current mode. At the inlet of the air channel, dry air $\left(\mathrm{N}_{2}: \mathrm{O}_{2}=79: 21\right)$ is supplied at a constant temperature of $550{ }^{\circ} \mathrm{C}$. The inlet velocity of the air flow is fixed at $1.84 \mathrm{~m} / \mathrm{s}$. It corresponds to the oxygen utilization factor of $5 \%$ in the discharge operation. Note that the air flow plays an important role in maintaining the system temperature within a certain limit. Because the air inlet temperature is kept constant, the air acts as coolant when there is excess heat generation in the system, while it acts as a heat supply when there is insufficient heat in the system.

\section{Results and Discussion}

\section{3-1. Fundamental Characteristics under Charge/Discharge Operations}

Figure 3 shows the operation curves, i.e., the terminal voltage as a function of capacity density, under charge/discharge conditions for various iron densities. The iron density is the amount of iron per unit area of the SOEC electrode. The thickness of the redox metal in the container, $L_{2}$, is proportional to the iron density. $L_{2}$ is $4.2,21$, 42 and $82 \mathrm{~mm}$ for iron density $1,5,10$ and $20 \mathrm{~g} / \mathrm{cm}^{2}$, respectively. Note that the 
capacity density is proportional to SOC or (1-SOC) since SOC is the normalized capacity. The capacity is also proportional to the elapsed time because the current density is kept constant, although each iron density has a different elapsed time. The operation time is approximately $6.5 \mathrm{~h}$ for an iron density of $1 \mathrm{~g} / \mathrm{cm}^{2}$.

Comparing the discharge and charge operations, the terminal voltage under the charge operation is naturally higher than that under the discharge operation. For each operation, sharp changes are observed in the last part above a capacity density of $1200 \mathrm{mAh} / \mathrm{g}-\mathrm{Fe}$. This reflects the limited reaction of the redox metal since there is little usable redox metal remaining at this stage. In the discharge operation, the terminal voltage is lower at a higher iron density for the same SOC. The situation is opposite in the charge operation; the terminal voltage is higher for a higher iron density. The difference of the terminal voltage for different iron density is related to its temperature and gas diffusion in the container, which are discussed in latter sections.

It was shown that the discharge and charge operations produce similar results, although their tendencies are almost opposite. Therefore, the results of the discharge operation are discussed first hereafter, followed by those of the charge operation, although graphs of both operations are shown next to each other in some figures.

\section{3-2. Heat Transfer during Discharge Operation}

In the discharge operation, the oxidation of iron and the power generation reactions in the SOEC are exothermic reactions generating heat. The generated heat is removed from the system by the convective heat transfer between the air flow and the SOEC. This means that the air functions not only as the oxygen supply for the electrochemical reaction but also as the coolant of the system. The thermal budget during the discharge operation is shown in Fig.4 (a) for an iron density of $1 \mathrm{~g} / \mathrm{cm}^{2}$. In the figure, a negative value implies heat removal from the system. Heat generation 
from the SOEC and the redox metal is almost constant under the constant-current operation adopted in this study. In contrast, the amount of heat exchanged between the battery and the air flow through convective heat transfer increases as time elapses. The figure shows that the cooling effect of the air flow is not sufficient particularly at the beginning of the operation.

The changes in the average temperatures of the SOEC and the redox metal corresponding to Fig.4 (a) are shown in Fig.5 (a). The average temperature rises by about $50{ }^{\circ} \mathrm{C}$ during the discharge operation. The temperature rise is steep at the beginning of the operation when the temperatures of the SOEC and the redox metal are relatively low. Since the air inlet temperature is kept constant in these calculations, the temperature difference between the air flow and the SOEC is relatively small at the beginning of the operation, which limits the cooling effect of the air. As a result, there is a large amount of excess heat generation that leads to a rapid temperature rise. As time elapses, the average temperature of the SOEC increases, increasing the temperature difference between the air flow and the SOEC. This enhances the cooling effect of the air as shown in Fig.4 (a) and the gradient in Fig. 5(a) becomes gradually small as time elapses.

\section{3-3. Current Density Distribution of SOEC during Discharge Operation}

The distributions of the SOEC local temperature, the oxygen partial pressure in the air flow, and the local current density along the air flow direction ( $x$-direction) are shown in Fig. 6 for an iron density of $1 \mathrm{~g} / \mathrm{cm}^{2}$ at SOCs of 2, 50, and 98\%. Fig. 6 (a) shows that the local temperature profile is relatively uniform, particularly at the beginning of the discharge operation (SOC $=98 \%$ ) and increases almost uniformly as time elapses. In the upstream region, where the SOEC is exposed to fresh air, temperature is relatively lower. The temperature difference along the $x$-direction is approximately $12{ }^{\circ} \mathrm{C}$ at $\mathrm{SOC}=2 \%$. The oxygen partial pressure decreases along the 
air flow direction, as shown in Fig. 6 (b), although the hydrogen partial pressure is almost uniform along the SOEC (not shown). The local current density is higher in the upstream region as shown in Fig. 6 (c). The slope of the local current density is steep in the upstream region for $\mathrm{SOC}=50 \%$ and $2 \%$. This is attributed to the above-mentioned temperature distributions. At high temperatures, the activation overpotential is lower and the system becomes more sensitive to the EMF. Because of the relatively low temperature in the upstream region, the EMF is higher in this region, resulting in a higher local current density. The difference in the local current density between the upstream and downstream is about $9 \%$. Although the local temperature profile is relatively uniform with a maximum difference of approximately $12{ }^{\circ} \mathrm{C}$ in this study, it has a clear effect on the local current density distribution.

\section{3-4. Evolution of Redox Reaction Area during Discharge Operation}

The hydrogen partial pressure during the discharge operation is shown in Fig.7 (a) for four cases with different iron densities. It is an average value of the hydrogen partial pressure in the free diffusion space between the SOEC and redox metal. As the temperature rises (Fig.5 (a)), the equilibrium partial pressure of hydrogen decreases since the metal oxidation reaction is exothermic (Eq. (3)). On the other hand, the hydrogen partial pressure must be slightly below the equilibrium pressure during the discharge operation so that the redox metal can supply sufficient hydrogen. Then, the hydrogen partial pressure decreases with time as shown in Fig. 7 (a), following the decrease in the equilibrium pressure. Note that the difference between the hydrogen partial pressure and the equilibrium pressure depends on the balance between the electrochemical reaction at the SOEC and the redox reaction. If the redox reaction is slow and the hydrogen generation rate is insufficient to replace the hydrogen consumed by the SOEC, the hydrogen partial pressure drops and the difference from the equilibrium pressure becomes large. This enhances the redox 
reaction to generate more hydrogen. In contrast, if the redox reaction is sufficiently fast, the hydrogen partial pressure remains close to the equilibrium pressure. These tendencies correspond well with those in another experiment that we performed (not published). At the end of the operation, when little fresh iron remains, the hydrogen partial pressure sharply drops as shown in Fig. 7 (a). This is consistent with the sharp drop in the terminal voltage observed in Fig.3 (a).

In Fig. 7 (a), the hydrogen partial pressure is generally lower for a higher iron density. It is a combined effect of the temperature and gas diffusion. In addition to the above mentioned change of the equilibrium pressure due to the temperature, the gas diffusion affects the gas composition near the SOEC and consequently, the EMF. To observe its effects, the distributions of the local hydrogen generation rate in the redox metal and the local SOC are first examined in this section for an iron density of 1 $\mathrm{g} / \mathrm{cm}^{2}$ as shown in Figs. 8 and 9, respectively. Note that only the redox metal region is depicted in these figures. Metal oxidation first proceeds actively near the surface approximately $3 \mathrm{~mm}<y<4 \mathrm{~mm}$ as shown in Fig. 8 (a). Then, reflecting the higher local current density in the upstream shown in Fig. 6 (c), the main active area is the upstream region of approximately $0 \mathrm{~mm}<x<20 \mathrm{~mm}$ (Fig. 8(b)). The local SOC shown in the corresponding figures (Figs. 9 (a) and (b)) indicates the ratio of fresh iron. It is clearly shown that after the fresh iron near the upstream surface region is consumed, the active area shifts to the bottom downstream region, where a large amount of iron remains (Figs. 8 (c) and 9 (c)). It makes the gas diffusion path in the redox metal longer. Because the diffusion resistance increases, the gas composition in the space between the SOEC and the redox metal shifts to steam-rich composition. The gas diffusion effect is discussed in the next session.

\section{3-5. Gas Diffusion during Discharge Operation}


Figure 10 shows the evolution of the distribution of local hydrogen partial pressure for an iron density of $1 \mathrm{~g} / \mathrm{cm}^{2}$. Note that this figure covers not only the redox metal region but also the space between the redox metal and the SOEC electrode. When the metal is oxidized, it expands and the local porosity decreases. The nonuniform local porosity and its evolution inevitably affect the effective gas diffusion coefficient in the porous metal region. These effects are taken into account in the calculations. At SOC $=98 \%$, hydrogen is generated near the surface of the redox metal as shown in Figs. 8 (a) and 10 (a). Because of the uniform hydrogen production in the $x$-direction, the gas diffusion can be approximated as 1-D diffusion in the positive $y$-direction. Note that because of the redox reaction, the local porosity of the redox metal becomes lower near the surface at the beginning of the discharge process. The low-porosity region formed near the surface acts as a barrier layer to reduced gas diffusion. At $\mathrm{SOC}=50 \%$, shown in Fig. 10 (b), the hydrogen generation is active in the upstream region. The generated hydrogen is supplied to the SOEC by diffusion while some of the hydrogen is trapped in the bottom region of the redox metal. This is because the resistance to diffusion near the redox surface is relatively high owing to the low porosity mentioned above. At SOC $=2 \%$, the hydrogen generation is only active in the bottom downstream region in the redox metal. The supply of hydrogen from only this region results in the contour map shown in Fig. 10 (c), which is very different from that in Fig. 10 (a). Reflecting the reduced gas diffusion in the redox metal region $(0 \mathrm{~mm}<y<4 \mathrm{~mm})$, the gradient is steep in this area. In Fig. 10(c), if we calculate the gradient in $y$-direction at $x=90 \mathrm{~mm}, y=3 \mathrm{~mm}$ (inside of the porous metal), its value is 7 times larger than that calculated at $x=90$ $\mathrm{mm}, y=5 \mathrm{~mm}$ (outside of the porous metal).

Although there is a clear difference in the gradient reflecting the difference of the effective diffusion coefficient, the distribution of the hydrogen partial pressure can generally be regarded as uniform for an iron density of $1 \mathrm{~g} / \mathrm{cm}^{2}$ shown in Fig. 10. Its 
non-uniformity increases when the iron density is higher because the redox metal thickness, $L_{2}$, is longer. The maximum difference of the hydrogen partial pressure observed at $\mathrm{SOC}=50 \%$ and $2 \%$ for iron density of $20 \mathrm{~g} / \mathrm{cm}^{2}$ was 0.025 and $0.043 \mathrm{~atm}$ that correspond to around 5 and $8 \mathrm{mV}$ of EMF, respectively. It means if the gas diffusion is effectively enhanced in the redox metal region, its terminal voltage shown in Fig. 3(a) can be improved to take a closer value of the iron density of $1 \mathrm{~g} / \mathrm{cm}^{2}$. The insufficient gas diffusion in the redox metal also causes another and probably severer problem of a non-uniform utilization of the redox metal. Its effect will be more prominent under high iron density condition and is to be discussed in section 3-7.

\section{3-6. Charge Operation}

The phenomena observed during the charge operation can generally be understood as the result of reverse reactions to those occurring in the discharge operation. As shown in Fig. 4 (b), the heat absorption resulting from the reduction of iron oxide and the electrochemical reaction is almost constant during the operation. Note that the SOEC operates below the thermal neutral point owing to the relatively low current density assumed in this study. The heat supplied by the air is insufficient to maintain the temperature of both the SOEC and the redox metal (Fig. 5(b)). Their average temperatures continue to decrease during the operation and never reach a thermal steady state. It is worth noting that the heat generation caused by the internal losses of the SOEC can be effectively utilized to the endothermic reactions during the

charge operation, not only for the steam electrolysis but also for the reduction of $\mathrm{Fe}_{3} \mathrm{O}_{4}$. If the current density is high and heat generation from the SOEC is sufficient, there will be no need for additional heat supply from outside of the system.

The distributions of the SOEC local temperature, the oxygen partial pressure in the air flow, and the local current density along the $x$-direction during the charge operation are shown in Fig. 11, which correspond to those in Fig. 6 for the discharge 
operation. There is a noticeable difference in the local current density distribution for the charge and discharge operations (Figs. 11 (c) and 6 (c)). In the charge operation, the local current density remains relatively flat. As the temperature decreases, a higher EMF is expected while the activation overpotential also increases. The current density distribution is determined as a result of the trade-off between these two effects.

The temporal change in the hydrogen partial pressure shown in Fig.7 (b) is consistent with the time course of the temperature shown in Fig. 5 (b). Figure 7 (b) also shows that the dependence of the hydrogen partial pressure on the iron density is similar to that in the discharge operation. The distributions of the local hydrogen absorption rate and local SOC are examined for an iron density of $1 \mathrm{~g} / \mathrm{cm}^{2}$ as shown in Figs. 12 and 13, respectively. Note that only the redox metal region is depicted in these figures. The active reaction area in the redox metal gradually shifts in a similar manner to that in the discharge operation. When iron oxide is reduced, it shrinks and the local porosity increases, increasing the rate of gas diffusion. This is a clear difference between the discharge operation and the charge operation as follows. In the discharge operation a barrier layer is formed near the surface region by the volume expansion of iron, while in the charge operation the gas diffusion is enhanced as the iron oxide is reduced. The active area moves to the inner region of the redox metal more rapidly in the charge operation. The evolution of the local SOC progresses almost uniformly as shown in Fig. 13 (b).

\section{3-7. Discharge/Charge Cycle Operation}

The discharge/charge cycle operation was calculated with iron densities of 1 and $5 \mathrm{~g} / \mathrm{cm}^{2}$. SOC is set at $100 \%$ at the beginning of the calculation. When SOC reaches $50 \%$ in the first discharge operation, the operation mode is switched to the charge operation and the battery is charged until SOC reaches $98 \%$. The discharge and charge operations are sequentially repeated three times with SOC varied between 
50 and $98 \%$. Figures 14 and 15 show the time courses of the temperature and SOC for iron densities of 1 and $5 \mathrm{~g} / \mathrm{cm}^{2}$, respectively. In the cycle operation, the temperature rises in the discharge operations and decreases in the subsequent charge operations as shown in Figs.14 (a) and 15 (a). As a result, the system temperature remains in a certain range without using any additional heating or cooling devices except for the air flow supplied at a constant temperature. During the cycle operation under calculation condition in this study, some of the heat released in the discharge operation is stored as a temperature increase in the battery itself and is utilized in the charge operation. In other words, the battery body is used as a thermal storage in this case.

One drawback of this configuration is the nonuniform utilization of the redox metal. Figures 14 (b) and 15 (b) show the time courses of the local SOC at three positions in the redox metal. When the iron density is $1 \mathrm{~g} / \mathrm{cm}^{2}$, as discussed in detail in the above sections, a difference in the local SOC is observed among the three positions. When the iron density is increased to $5 \mathrm{~g} / \mathrm{cm}^{2}$, this difference in the local SOC is significantly increased. The SOC of iron near the surface oscillates between 100 and $0 \%$, while that of the inner region always remains above $90 \%$. The iron particles near the surface are subjected to redox cycles involving large changes in SOC that may enhance degradation. As discussed in section 3-5, the gas diffusion in the redox metal is reduced particularly at low local SOC region. By enhancing the gas diffusion in the redox metal, e.g. use of pore former etc., difference in the local SOC observed in Fig. 15(b) is expected to be decreased. Careful design is needed to achieve a more uniform reaction of the redox metal, particularly for a battery with larger capacity.

\section{Conclusions}


A time-dependent 2-D numerical simulation was performed on an SOIAB to reveal the fundamental characteristics of this new system. A simple battery configuration was employed assuming a system with a small capacity. A simulation model for a unit element was developed considering heat and mass transfer in the system, taking both electrochemical and redox reactions into account. Particular attention was paid to the distributions of the local temperature, the concentrations of participating gas species, the reaction rate, the SOC, and the current density of the SOEC.

The numerical results showed the spatial and temporal changes in the temperature field in the charge and discharge operations, which were due to the combined effects of heat generation/absorption by the electrochemical and redox reactions and heat exchange with the air supplied through convective heat transfer. It was found that the system temperature continues increasing during the discharge operation and decreasing during the charge operation under the calculation conditions in this study. This is because the heat generation/absorption by the reactions is greater than the cooling/heating effect due to air convection. As the reaction rates are functions of the local temperature, in this system, thermal management will be a key issue to improve the battery performance.

It is possible to maintain the system temperature within a certain range by sequentially operating the battery in the discharge and charge modes since the heat released from the exothermic reactions in the discharge operation can be utilized for the endothermic reactions in the charge operation by using the battery itself as a thermal storage. It was also shown that there is a close relationship between the current density distribution in the SOEC and the evolution of the active reaction area in the redox metal. The active reaction area proceeds from the surface to the inner region and from upstream to downstream, reflecting the local porosity distribution and 
the tendency for a high current density in the upstream. This shows that the appropriate gas diffusion is also important for full and effective battery utilization. 


\section{REFERENCES}

[1] K. M. Abraham and Z. Jiang, J. Electrochem. Soc., 143 (1996), 1-5.

[2] A. K. Thapa and T. Ishihara, J. Power Sources, 196 (2011), 7016-7020.

[3] B. Dunn, H. Kamath, and J. M. Tarascom, Science, 334 (2011), 928-935.

[4] S. J. Skinner and J. A. Kilner, Solid State Ionics, 135 (2000), 709-712.

[5] N. Jayalakshmi and V. S. Muralidharan, J. Power Sources, 32 (1990), 277-286.

[6] N. Xu, X. Li, X. Zhao, J. B. Goodenough, K. Huang, Energy Environ. Sci. 4 (2011), 4942-4946.

[7] X. Zhao, Y. Gong, X. Li, N. Xu, and K. Huang, J. Electrochem. Soc., 160 (2013), A1241-A1247.

[8] X. Zhao, N. Xu, X. Li, Y. Gong, and K. Huang, ECS.Trans., 50 (2013), 115-123.

[9] M. Guo, X. Zhao, R. E. White, and K. Huang, J. Electrochem. Soc., 160 (2013), A2085-A2092.

[10] A. Inoishi, S. Ida, S. Uratani, T. Okano, and T. Ishihara, Phys. Chem. Chem. Phys., 14 (2012), 12818-12822.

[11] A. Inoishi, Y. W. Ju, S. Ida, and T. Ishihara, J. Power Sources, 229 (2013), $12-15$.

[12] A. Inoishi, Y. Okamoto, Y. W. Ju, S. Ida, and T. Ishihara, RSC Adv., 3 (2013), $8820-8825$

[13] H. Landes and R. Reichenbacher, ECS.Trans., 50 (2013), 47-68.

[14] W. Drenckhahn, H. Greiner, M. Kuhne, H. Landes, A. Leonide, K. Litzinger, C. Lu, C. Schuh, J. Shull, and T. Soller, ECS.Trans., 50 (2013), 125-135.

[15] N. H. Menzler, A. Hospach, L. Niewolak, M. Bram, O. Tokariev, C. Berger, P. Orzessek, W. J. Quadakkers, Q. Fang and H. P. Buchkremer, ECS.Trans., 57 (2013), 255-267.

[16] H. Ohmori, S. Uratani, and H. Iwai, J. Power Sources, 208 (2012), 383-390. 
[17] H. Iwai, H. Ohmori, K. Itakura, M. Saito, and H. Yoshida, ECS Trans., 57 (2013), 2661-2670.

[18] R. R. Melkote and K. F. Jensen, AIChE J., 38 (1992), 56-66.

[19] NIST Chemistry WebBook http://webbook.nist.gov/chemistry/.

[20] T. Suzuki, Z. Hasan, Y. Funahashi, T. Yamaguchi, Y. Fujishiro, and M. Awano, Science, 325 (2009), 852-855.

[21] T. Kawada, N. Sakai, H. Yokokawa, M. Dokiyo, M. Mori, and T. Iwata, J. Electrochem. Soc., 137, 3042-3047 (1990). 


\section{Captions}

Figure 1. Reactions in (a) discharge operation and (b) charge operation.

Figure 2. Schematic figure of the battery including the SOEC and the redox metal.

Figure 3. Operation curves in (a) discharge operation and (b) charge operation with iron densities of $1,5,10$, and $20 \mathrm{~g} / \mathrm{cm}^{2}$.

Figure 4. Heat generation balance of the system including both reaction heat and convectional heat in (a) discharge operation and (b) charge operation with iron density of $1 \mathrm{~g} / \mathrm{cm}^{2}$.

Figure 5. Temperature changes in (a) discharge operation and (b) charge operation with iron density of $1 \mathrm{~g} / \mathrm{cm}^{2}$.

Figure 6. Distributions along air flow of (a) temperature of SOEC, (b) oxygen partial pressure in air flow, and (c) current density of SOEC in discharge operation with iron density of $1 \mathrm{~g} / \mathrm{cm}^{2}$.

Figure 7. Change in hydrogen partial pressure in (a) discharge operation and (b) charge operation with iron densities of $1,5,10$, and $20 \mathrm{~g} / \mathrm{cm}^{2}$.

Figure 8. Distributions of hydrogen generation rate in the redox metal region in discharge operation at (a) $\mathrm{SOC}=98 \%$, (b) $\mathrm{SOC}=50 \%$, and (c) $\mathrm{SOC}=2 \%$ with iron density of $1 \mathrm{~g} / \mathrm{cm}^{2}$.

Figure 9. Distributions of local SOC in the redox metal region in discharge operation at (a) $\mathrm{SOC}=98 \%$, (b) SOC $=50 \%$, and (c) at SOC $=2 \%$ with iron density of $1 \mathrm{~g} / \mathrm{cm}^{2}$. Figure 10. Distribution of hydrogen partial pressure in the container in discharge operation at (a) SOC $=98 \%$, (b) SOC $=50 \%$, and (c) at $\mathrm{SOC}=2 \%$ with iron density of $1 \mathrm{~g} / \mathrm{cm}^{2}$.

Figure 11. Distributions along air flow of (a) temperature of SOEC, (b) oxygen partial pressure in air flow, and (c) current density of SOEC in charge operation with iron density of $1 \mathrm{~g} / \mathrm{cm}^{2}$. 
Figure 12. Distributions of hydrogen absorption rate in the redox metal region in charge operation (a) at $\mathrm{SOC}=2 \%$, (b) at $\mathrm{SOC}=50 \%$, and (b) at $\mathrm{SOC}=98 \%$ with iron density of $1 \mathrm{~g} / \mathrm{cm}^{2}$.

Figure 13. Distributions of local SOC in the redox metal region in charge operation (a) at $\mathrm{SOC}=2 \%$, (b) at $\mathrm{SOC}=50 \%$, and (b) at $\mathrm{SOC}=98 \%$ with iron density of 1 $\mathrm{g} / \mathrm{cm}^{2}$.

Figure 14. Time histories of (a) temperature and (b) local SOC during three-repeated discharge/charge cycle operation with iron density of $1 \mathrm{~g} / \mathrm{cm}^{2}$.

Figure 15. Time histories of (a) temperature and (b) local SOC during three-repeated discharge/charge cycle operation with iron density of $5 \mathrm{~g} / \mathrm{cm}^{2}$. 


\section{Tables}

Table 1. Mass generation rates of species by the electrochemical reactions $\left[\mathrm{kg}^{\circ} \mathrm{m}^{-3}\right.$ s-1].

\begin{tabular}{cccc}
\hline \hline \multirow{2}{*}{ Position } & \multirow{2}{*}{$\begin{array}{c}\text { SOEC } \\
\text { Operation }\end{array}$} & \multicolumn{2}{c}{$S_{\text {mass }, j}$} \\
\cline { 2 - 4 } & Discharge & $-\frac{M_{\mathrm{H}_{2}}}{2 F} \frac{i}{\Delta y}$ & $\frac{M_{\mathrm{H}_{2} \mathrm{O}}}{2 F} \frac{i}{\Delta y}$ \\
\hline $\begin{array}{c}\text { Computational grid } \\
\text { adjacent to fuel-side } \\
\text { electrode of SOEC }\end{array}$ & Charge & $\frac{M_{\mathrm{H}_{2}}}{2 F} \frac{i}{\Delta y}$ & $-\frac{M_{\mathrm{H}_{2} \mathrm{O}}}{2 F} \frac{i}{\Delta y}$ \\
\cline { 2 - 3 } & &
\end{tabular}

$\Delta y$ : Grid size in $y$ direction, $M_{j}$ : Molecular weight of species

Table 2. Boundary conditions.

\begin{tabular}{ccc}
\hline \hline $\begin{array}{c}\text { Fuel-side electrode of } \\
\text { SOEC }\end{array}$ & $\left.\lambda_{\text {gas }} \frac{\partial T_{\text {gas }}}{\partial y}\right|_{\text {interface }}=\left.\lambda_{\text {solid }} \frac{\partial T_{\text {solid }}}{\partial y}\right|_{\text {interface }}$ & $\frac{\partial Y_{j}}{\partial y}=0$ \\
\hline $\begin{array}{c}\text { Side walls } \\
(x=0,100 \mathrm{~mm})\end{array}$ & $\frac{\partial T}{\partial x}=0$ & $\frac{\partial Y_{j}}{\partial x}=0$ \\
\hline $\begin{array}{c}\text { Bottom wall } \\
(y=0 \mathrm{~mm})\end{array}$ & $\frac{\partial T}{\partial y}=0$ & $\frac{\partial Y_{j}}{\partial y}=0$ \\
\hline
\end{tabular}




\section{Acknowledgement}

This study was partially supported by Japan Science and Technology Agency (JST) /

Advanced Low Carbon Technology R\&D Program (ALCA). The authors also acknowledge Prof. Motohiro Saito, Mr. Tetsushi Isomoto and Mr. Kota Miyoshi of Department of Aeronautics and Astronautics, Kyoto University, and Prof. Masahiro Kishida of Department of Chemical Engineering, Kyushu University for their technical support and discussion. 


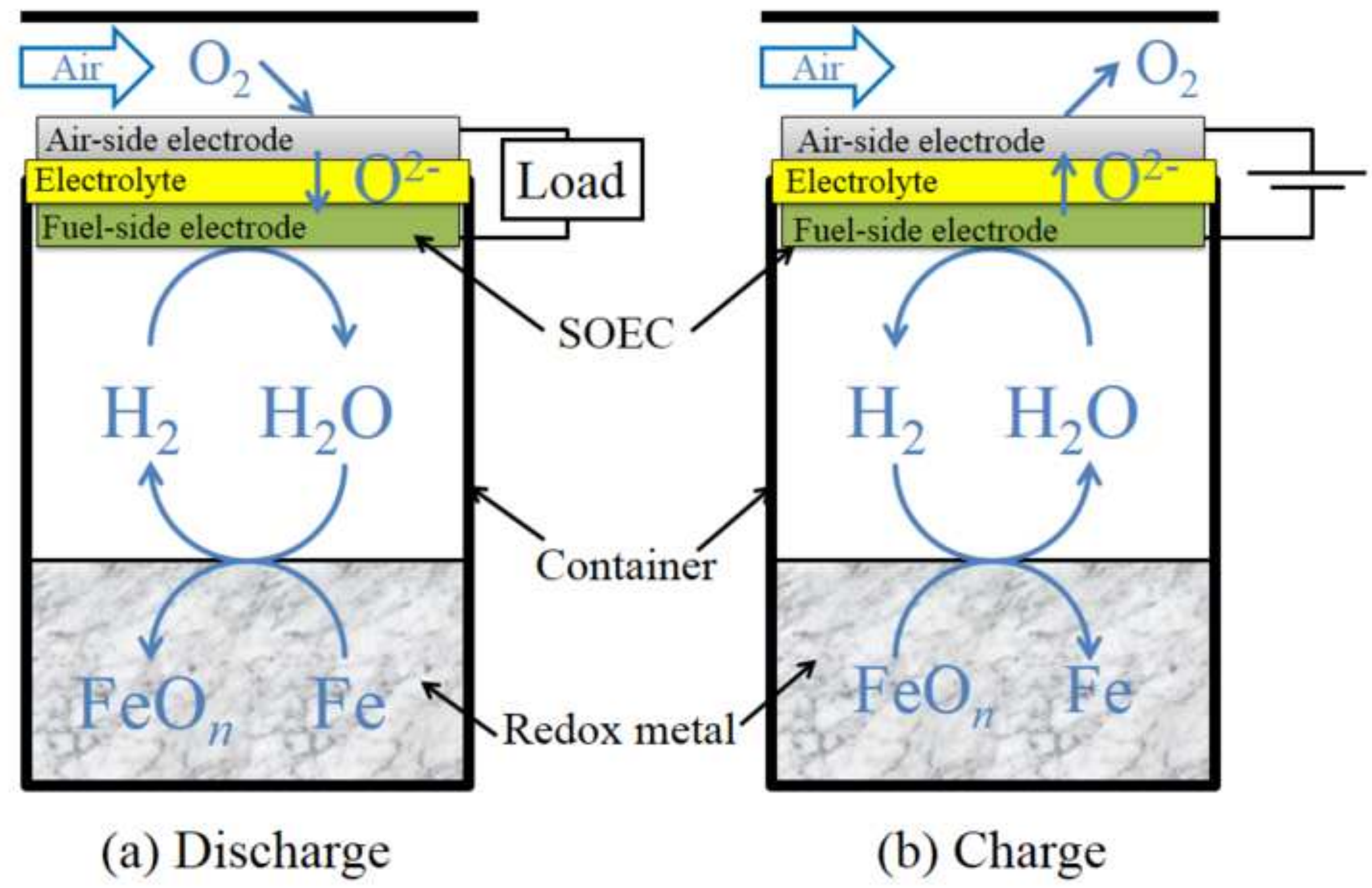

(a) Discharge (b) Charge 
Computational domain

Blue broken line: 1D

Red broken line: 2D

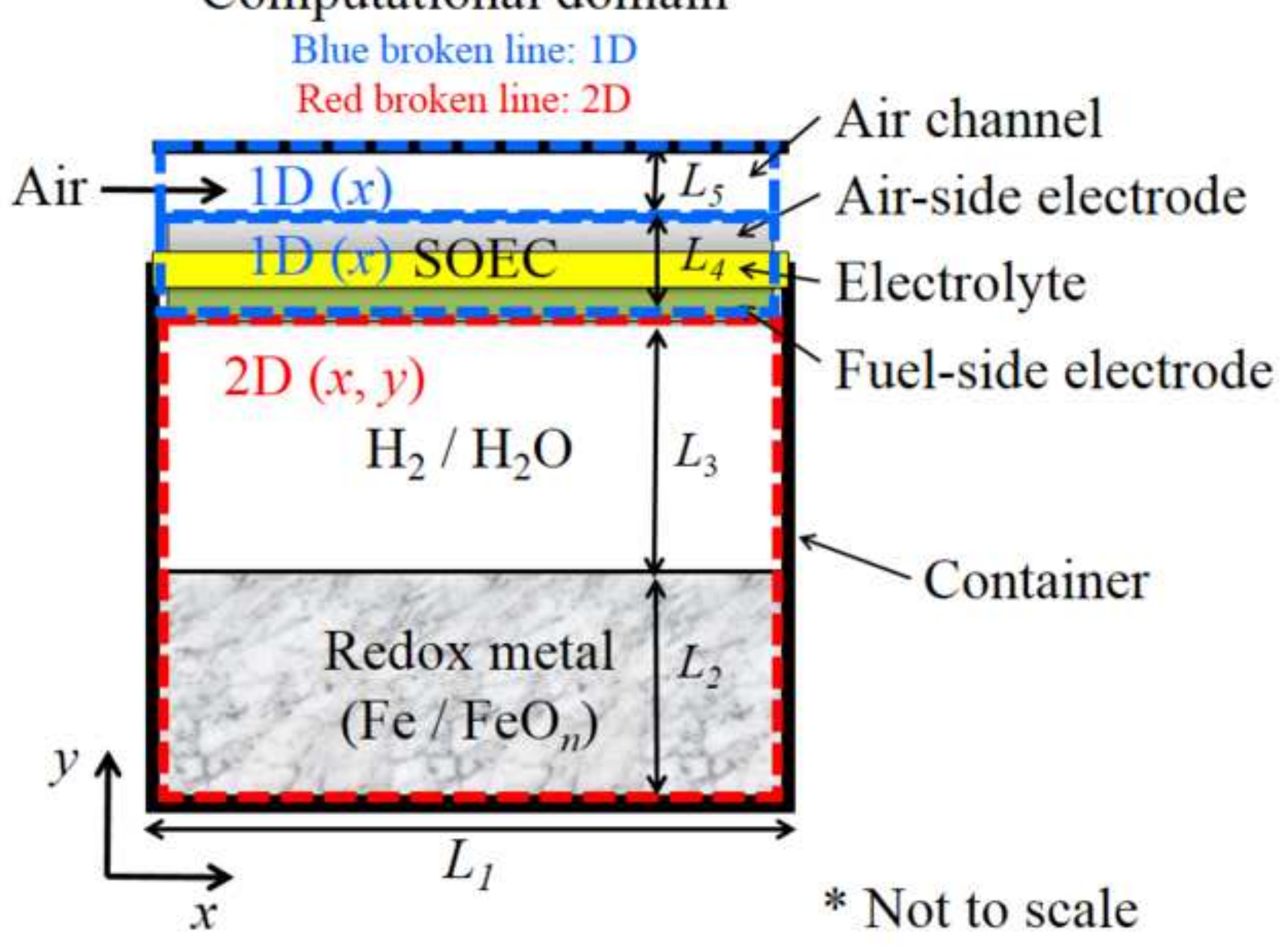




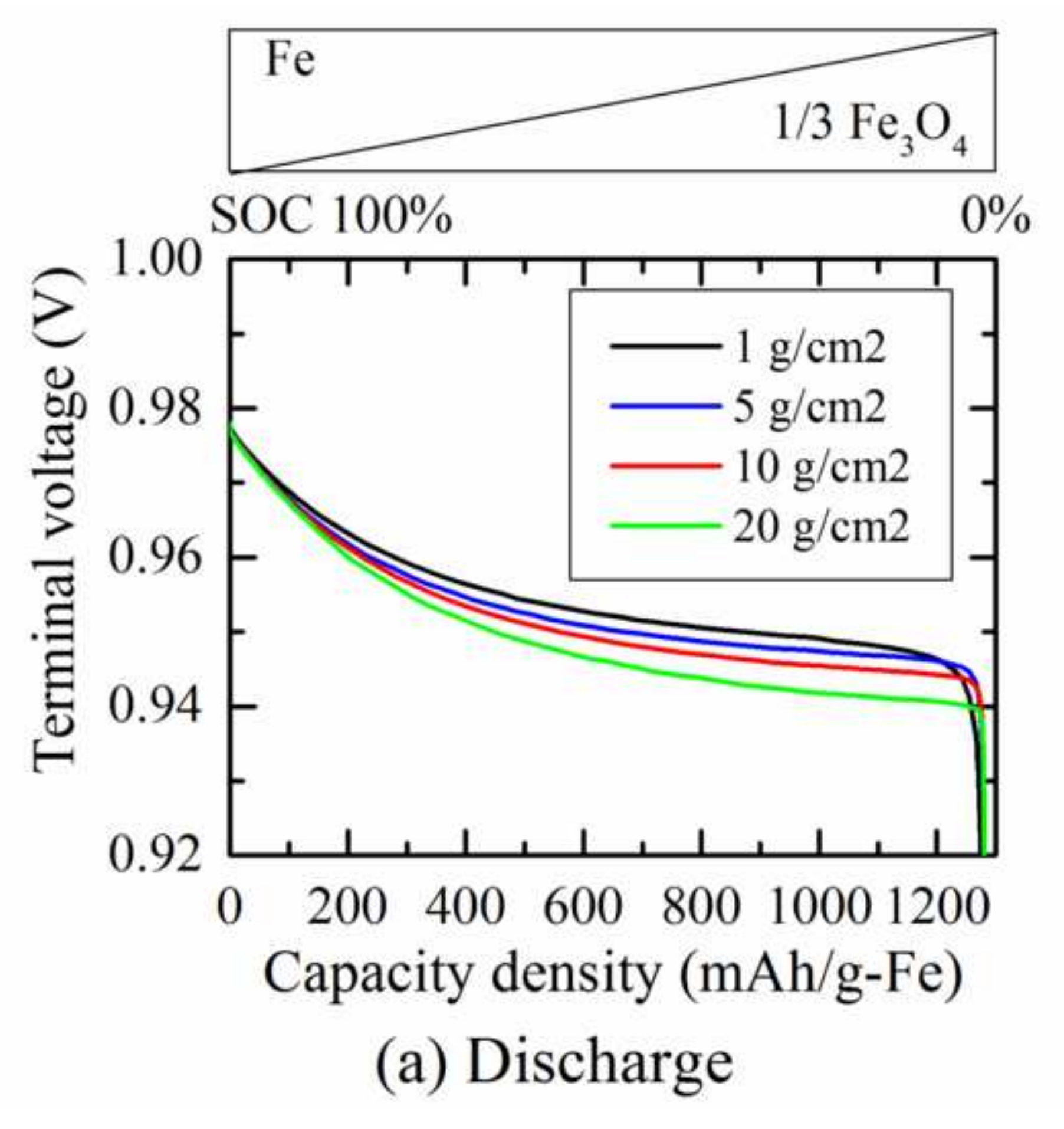

Figure.3a

$1 / 3 \mathrm{Fe}_{3} \mathrm{O}_{4}$

$0 \%$

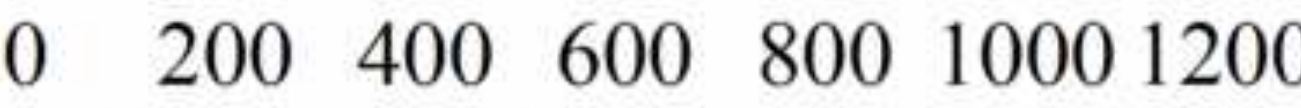

$\mathrm{Fe}$

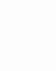
.
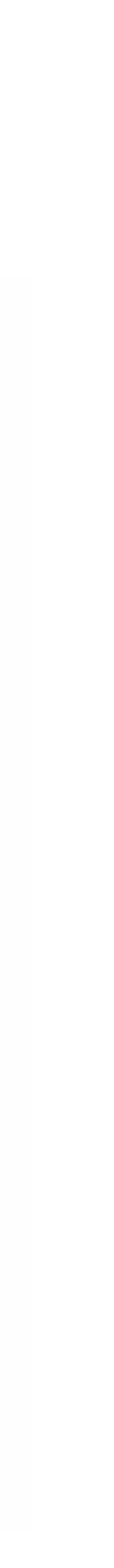

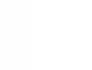
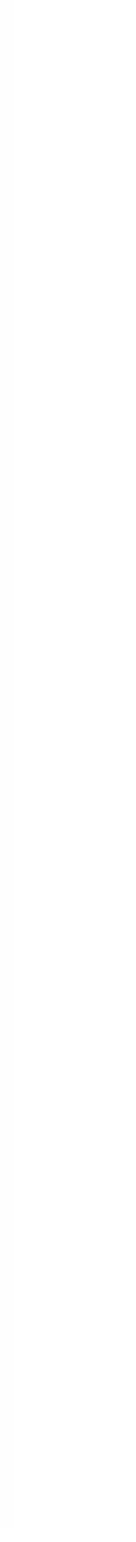


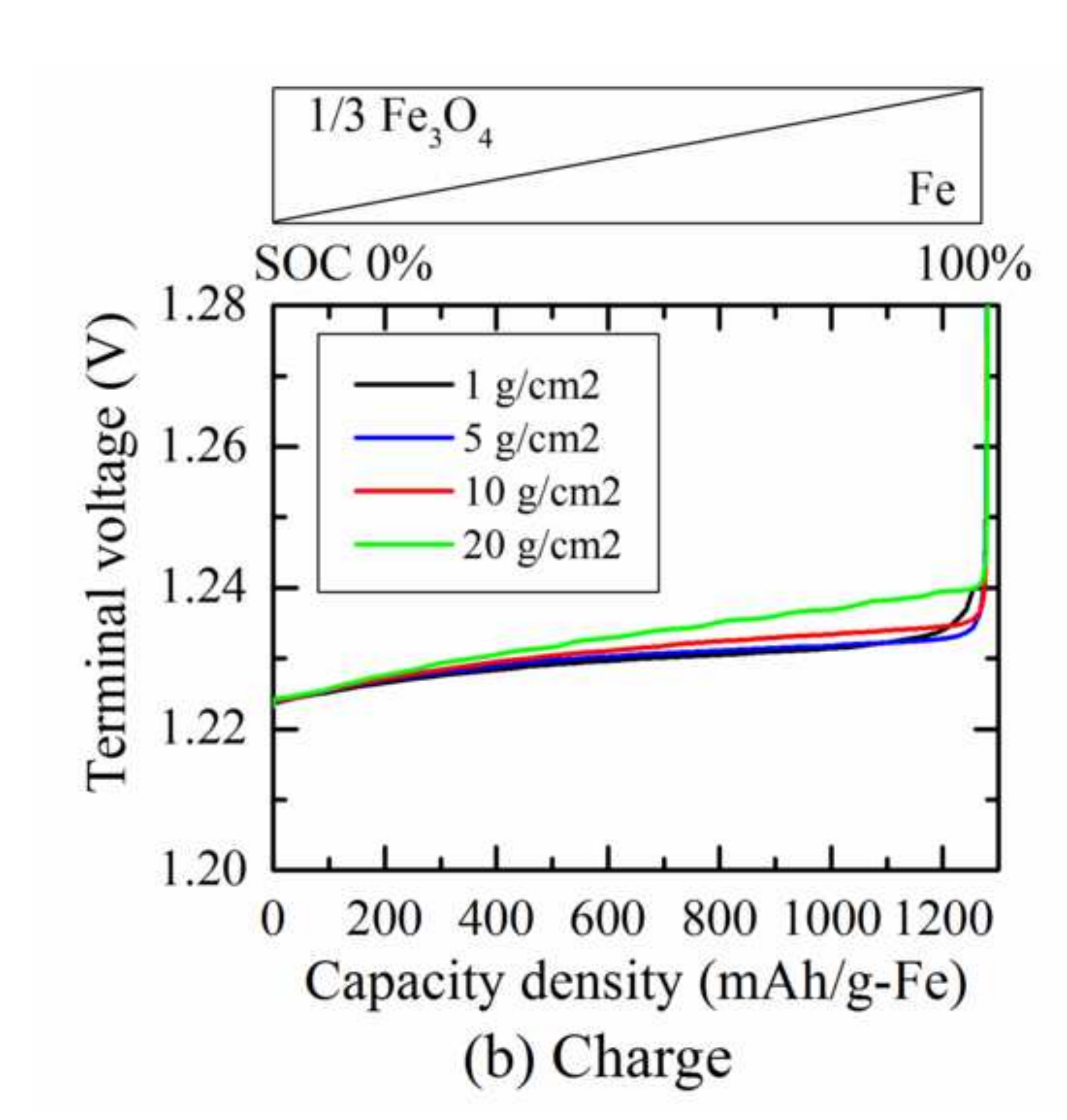

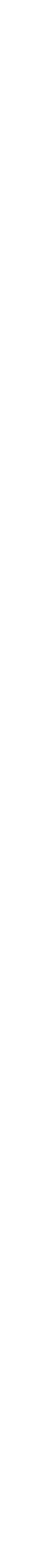

.
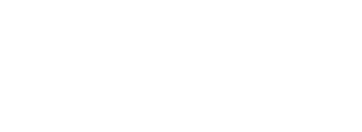

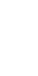

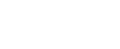

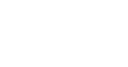

$-$

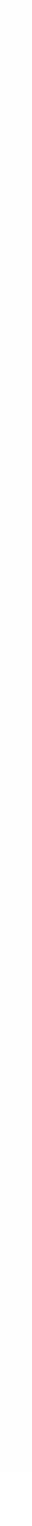




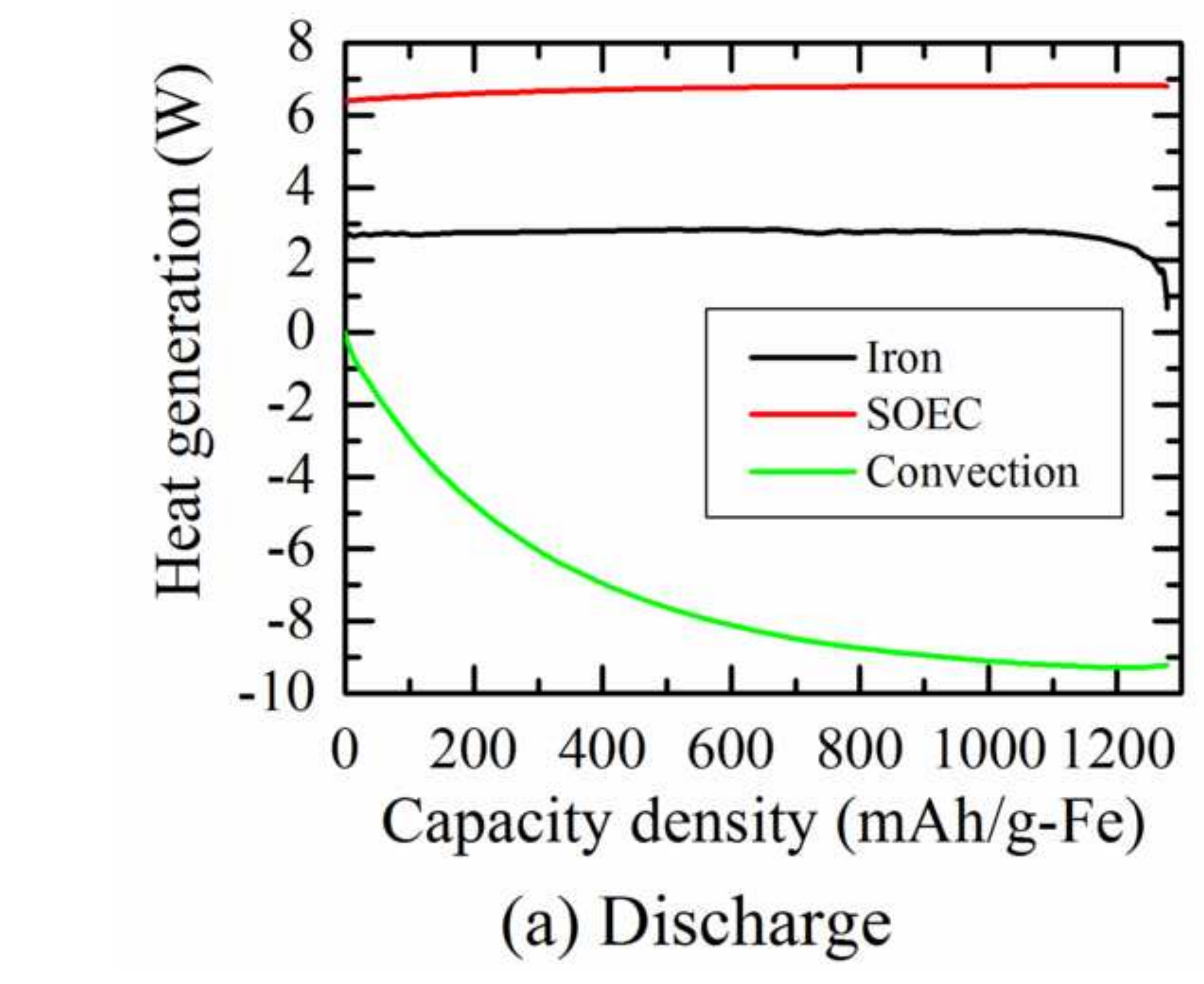

(a) Discharge 


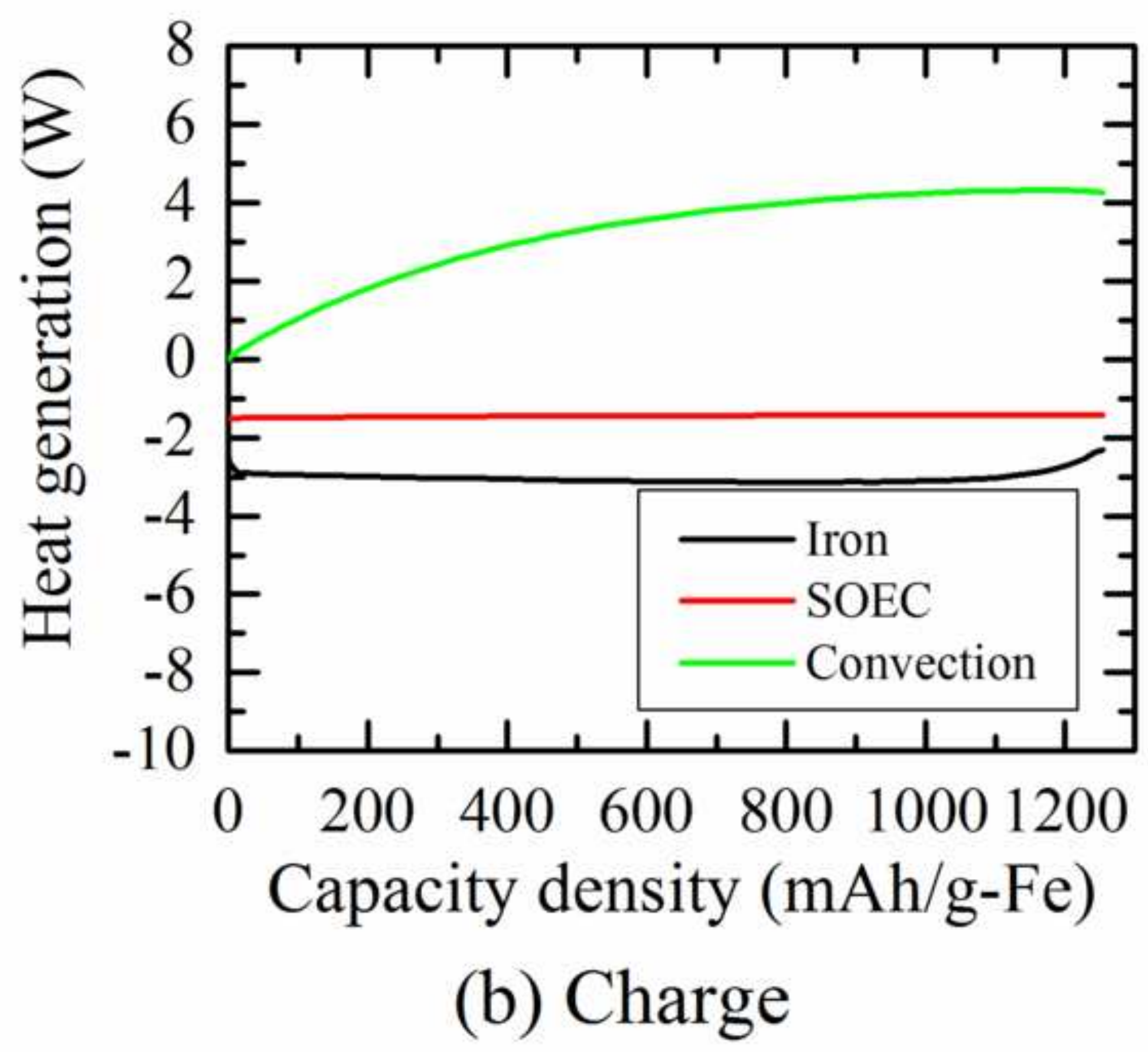

(b) Charge 


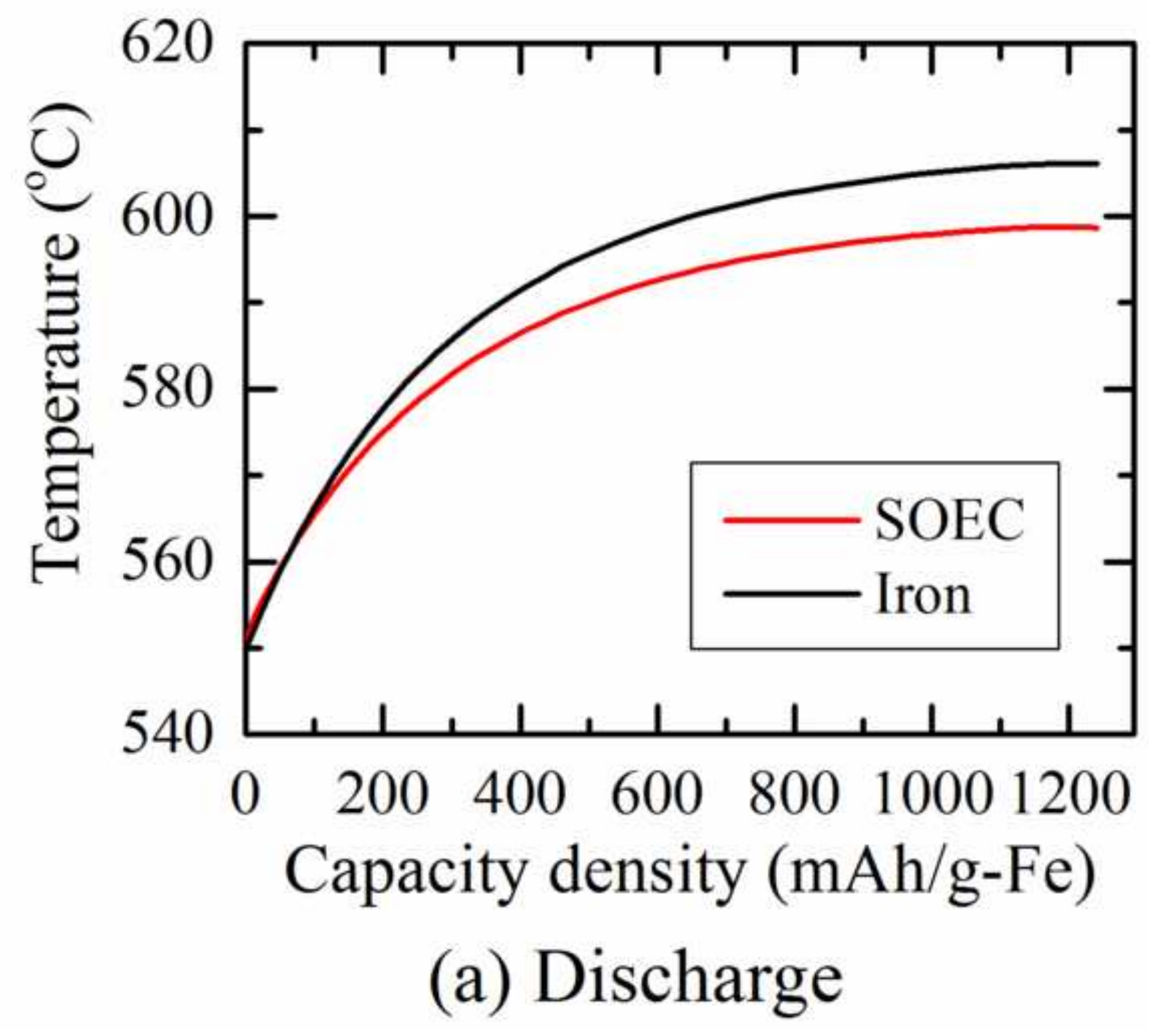




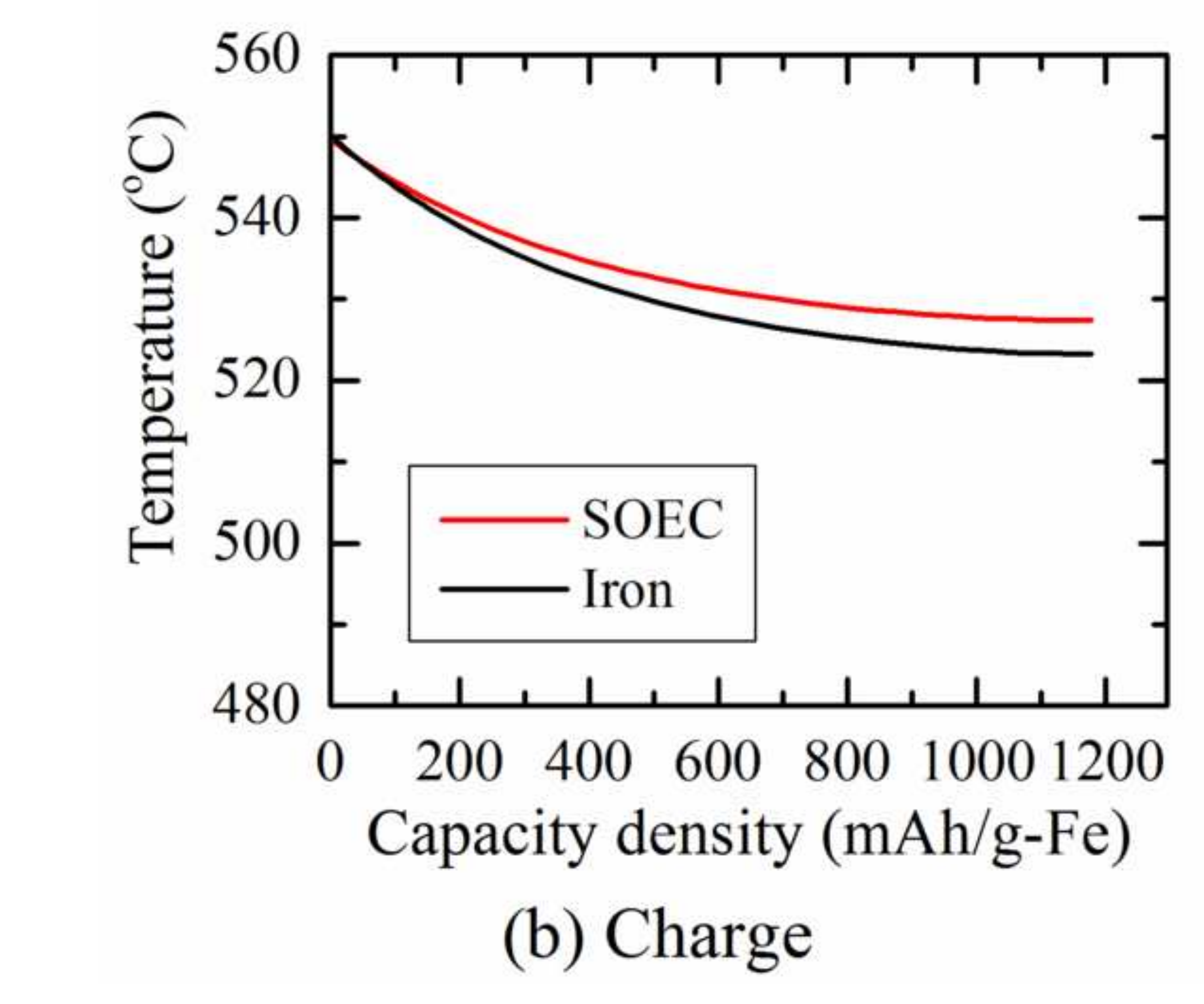




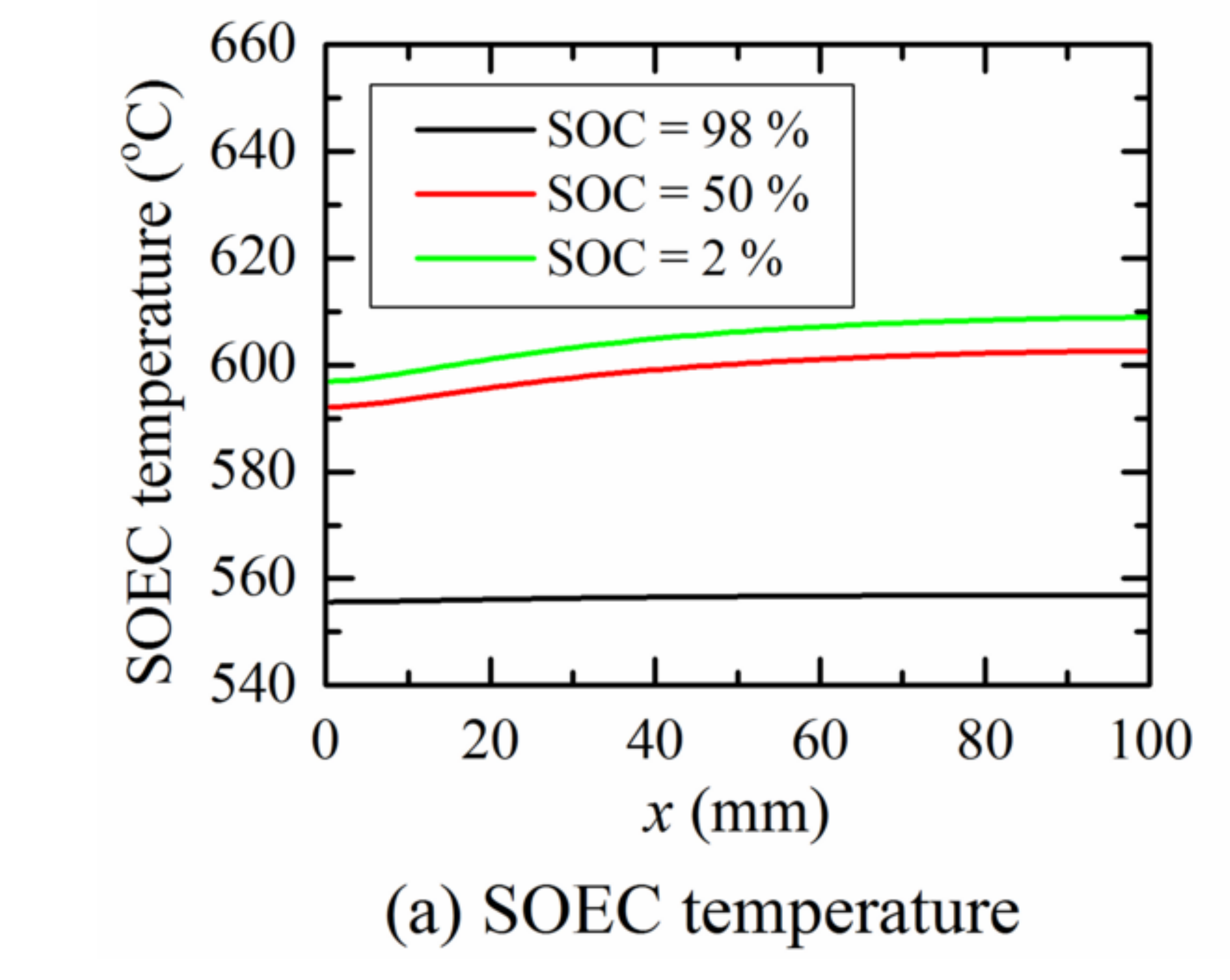




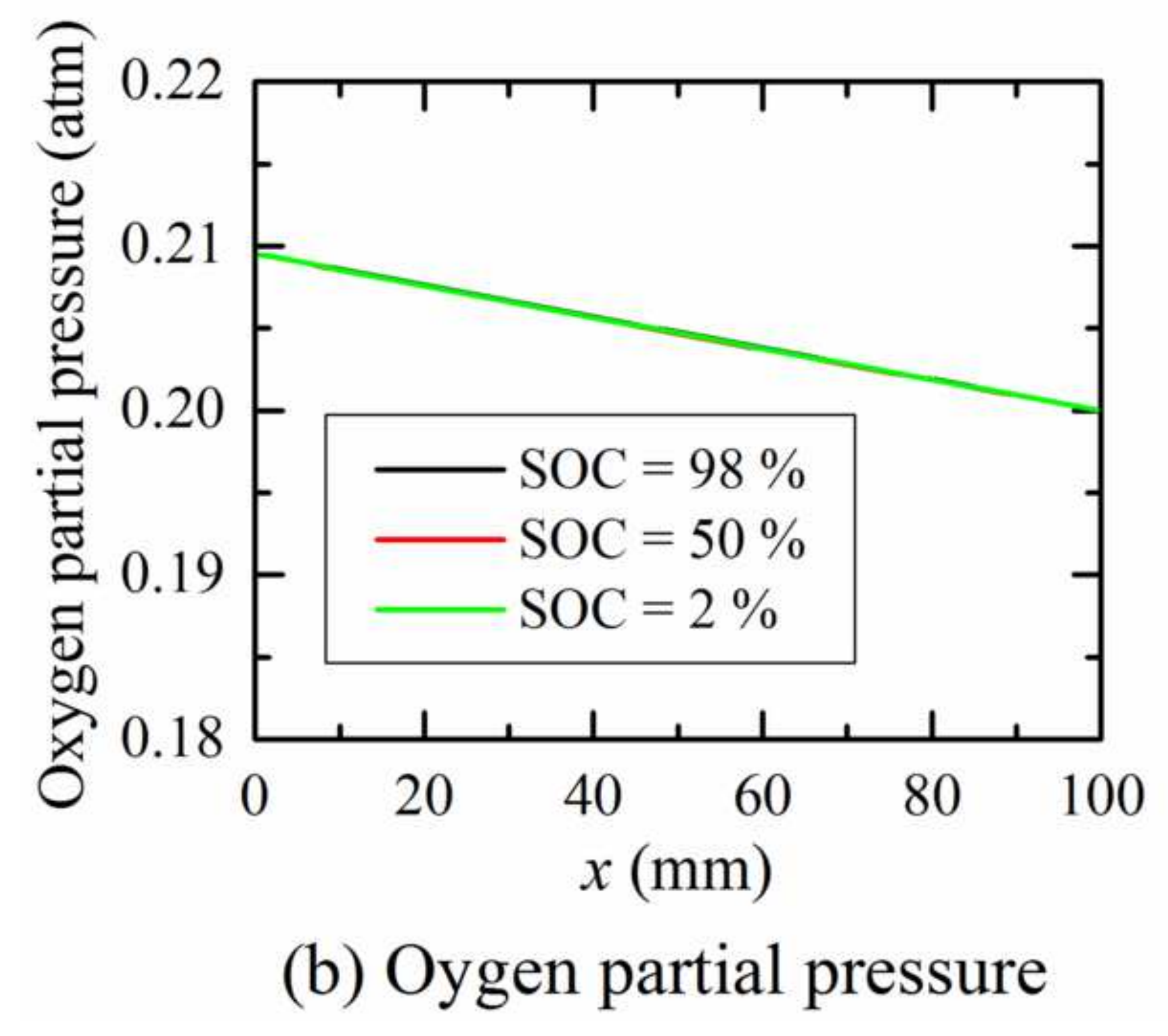

(b) Oygen partial pressure 


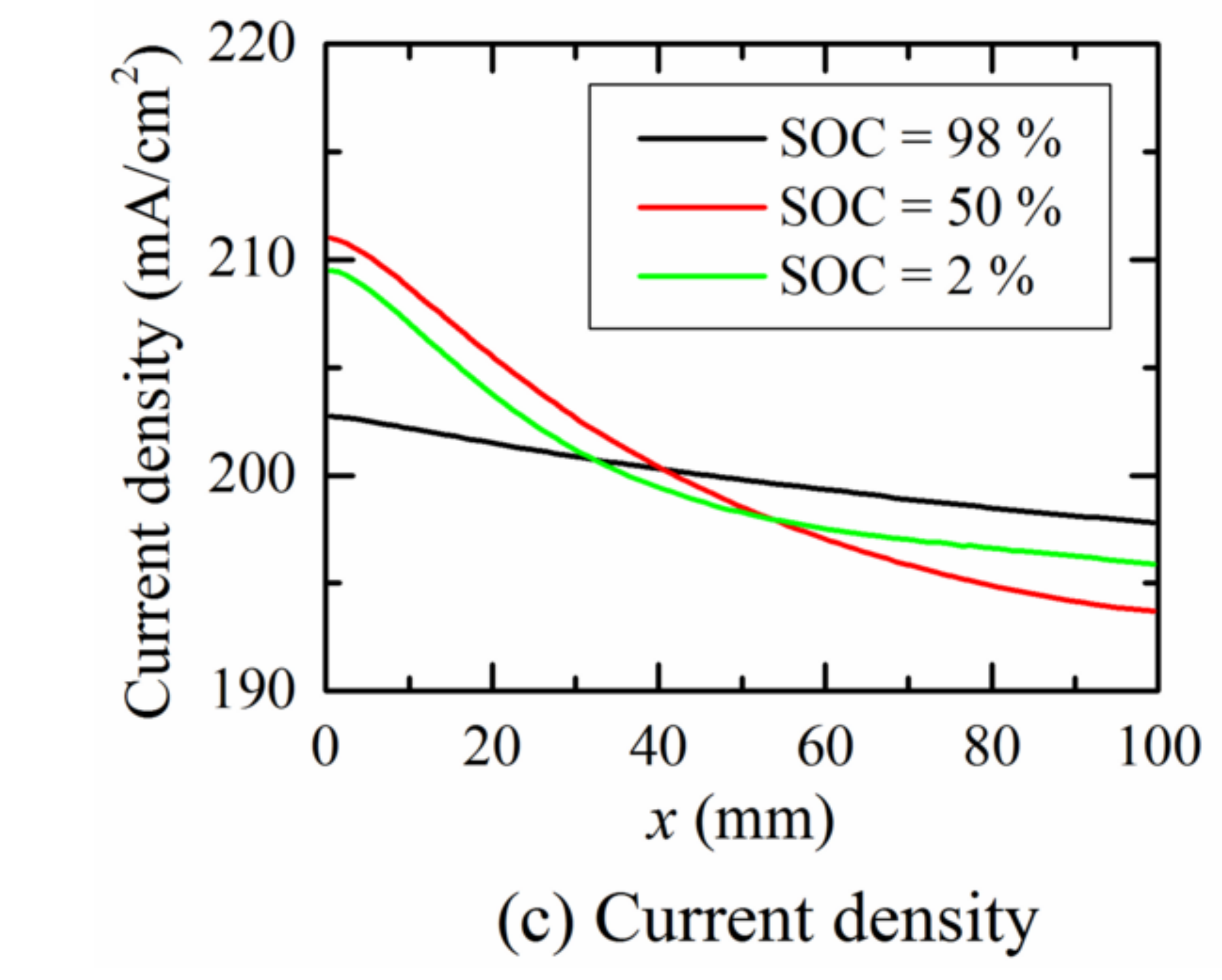

(c) Current density

(1)

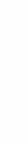

(1)




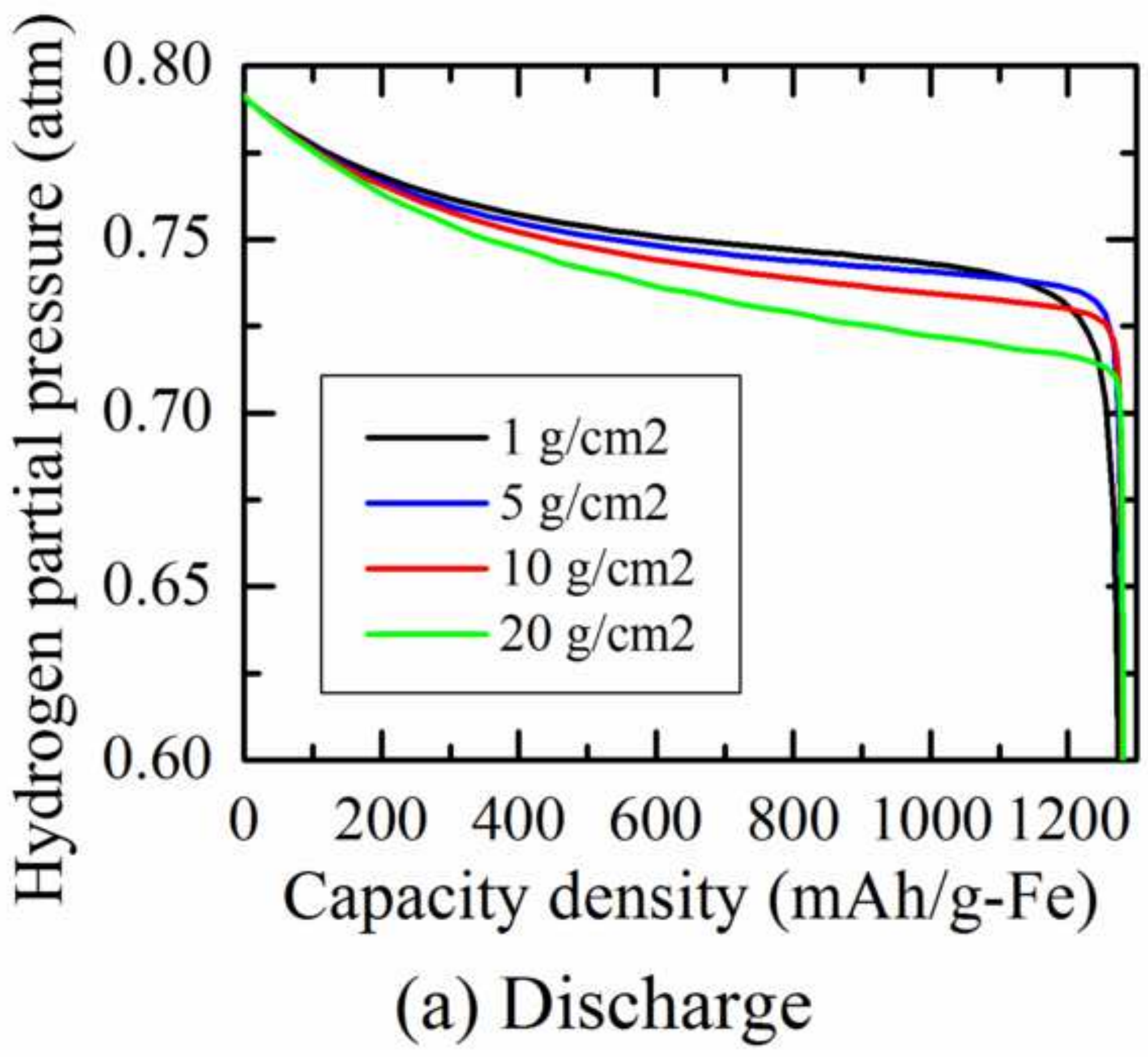




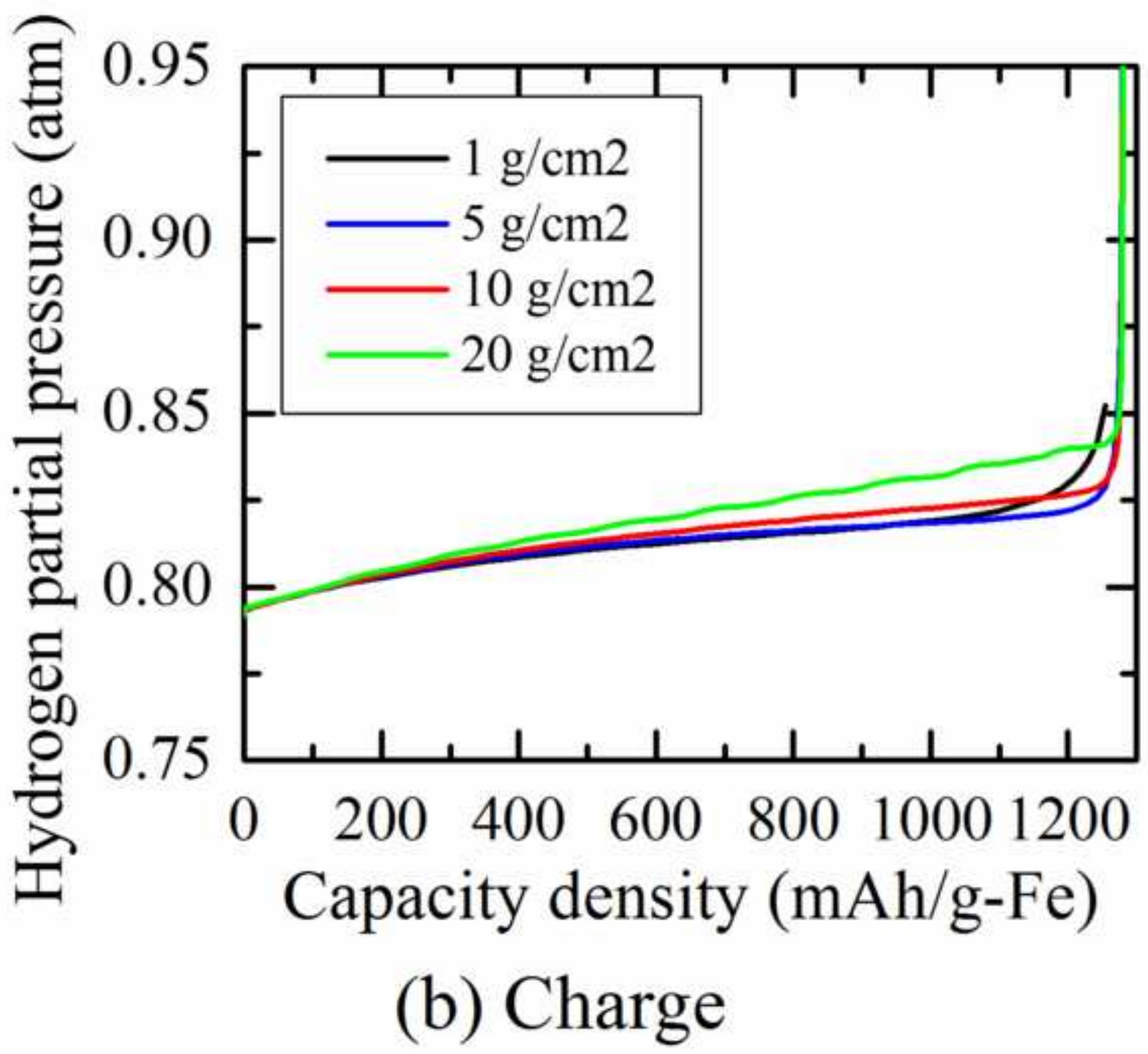




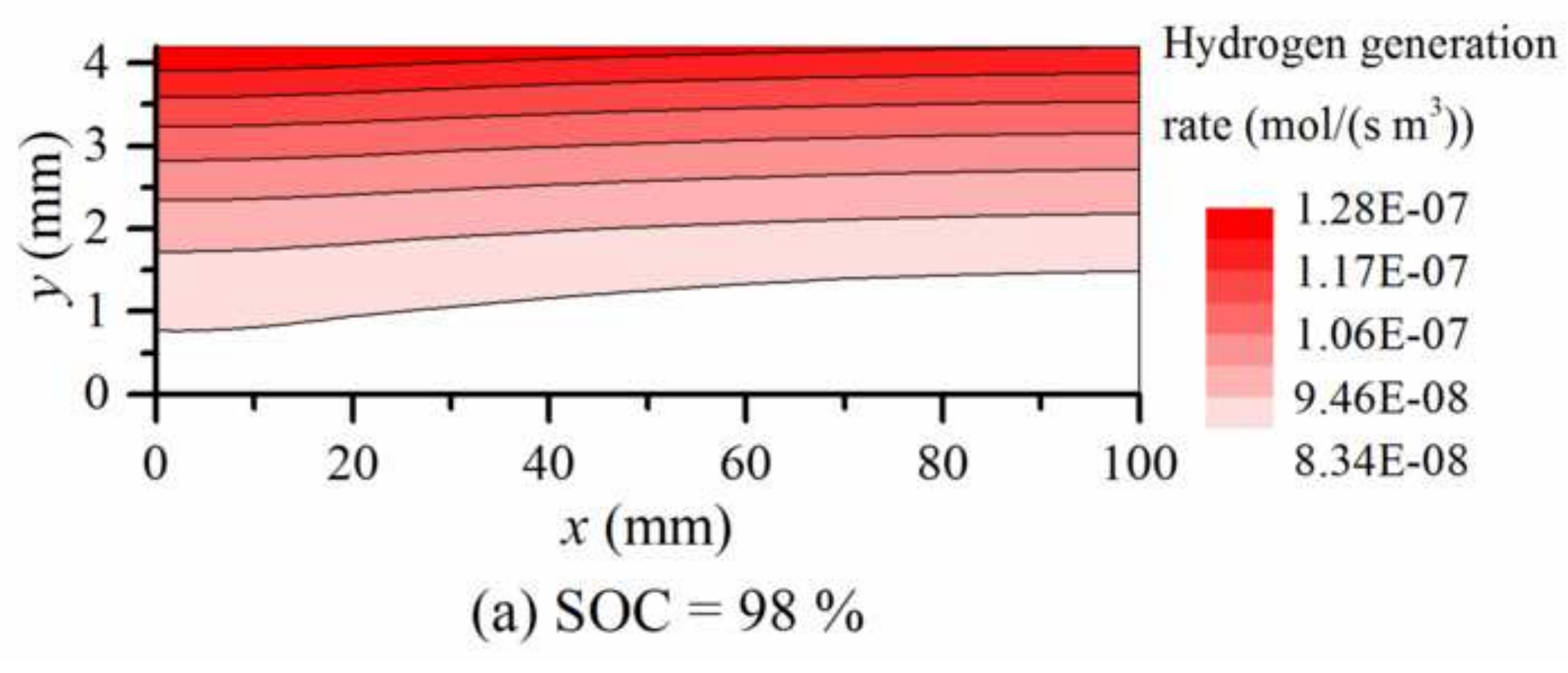

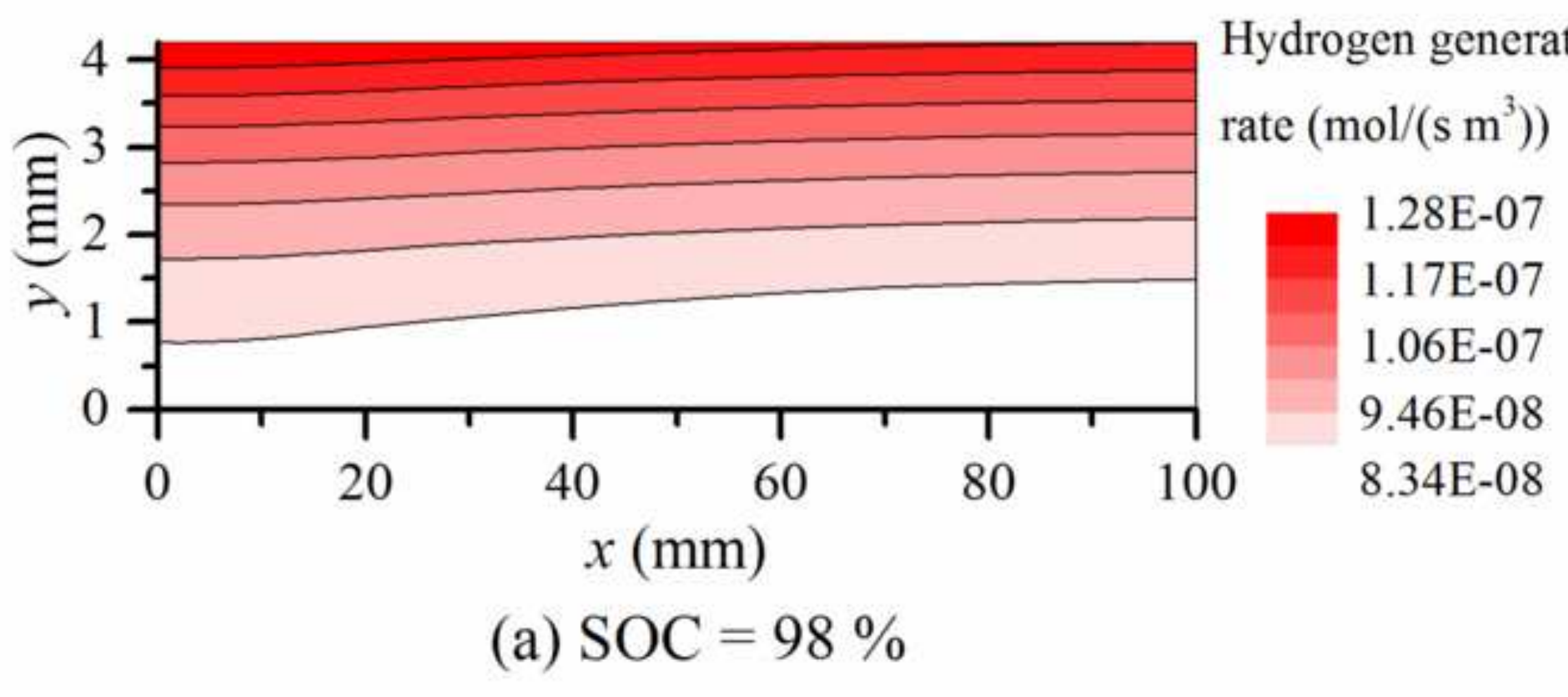

Hydrogen generation rate $\left(\mathrm{mol} /\left(\mathrm{s} \mathrm{m}^{3}\right)\right)$

1.28E-07

(a) $\mathrm{SOC}=98 \%$

\subsection{E-07 \\ $1.06 \mathrm{E}-07$ \\ 9.46E-08
$8.34 \mathrm{E}-08$ \\ 9.46E-08
$8.34 \mathrm{E}-08$ \\ $1.06 \mathrm{E}-07$}




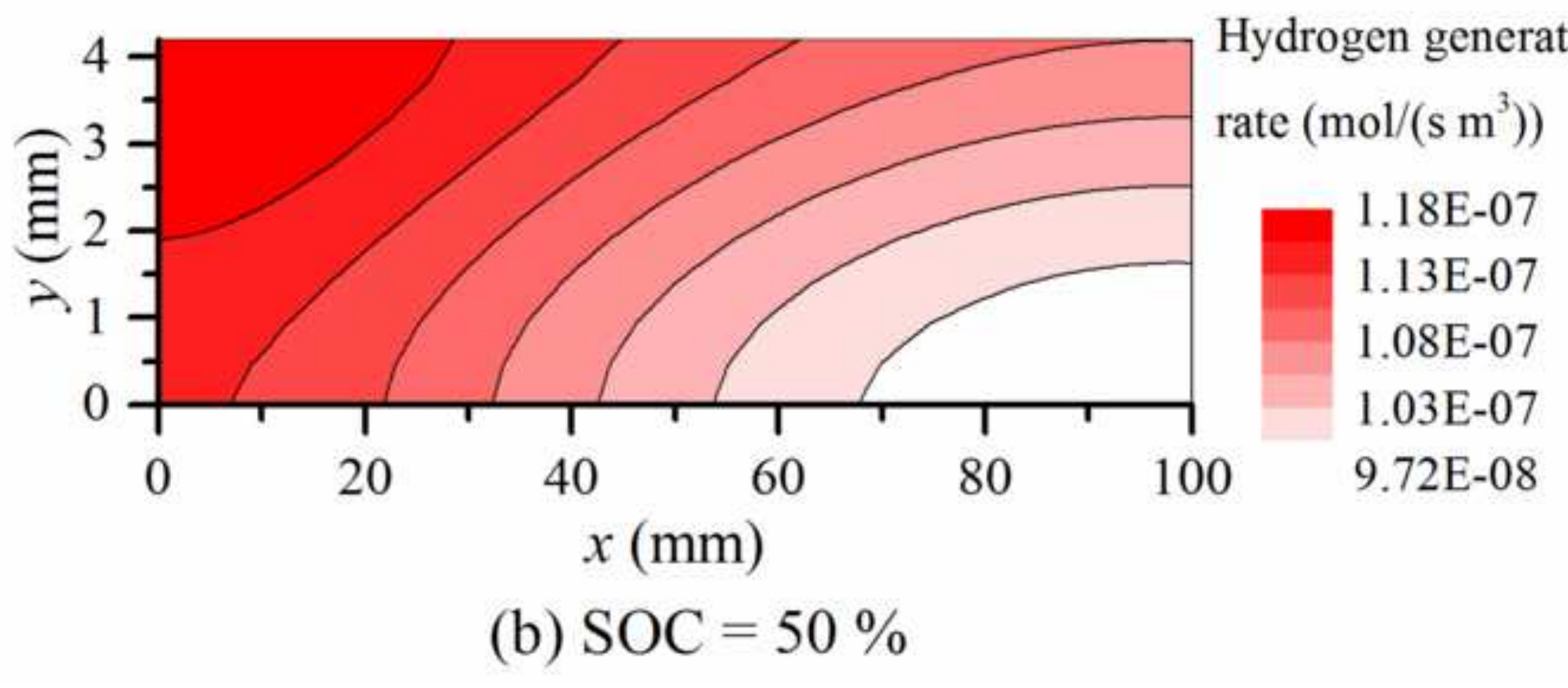

Hydrogen generation rate $\left(\mathrm{mol} /\left(\mathrm{s} \mathrm{m}^{3}\right)\right)$ 1.13E-07 $1.08 \mathrm{E}-07$ 1.03E-07 $9.72 \mathrm{E}-08$ (b) $\mathrm{SOC}=50 \%$ 


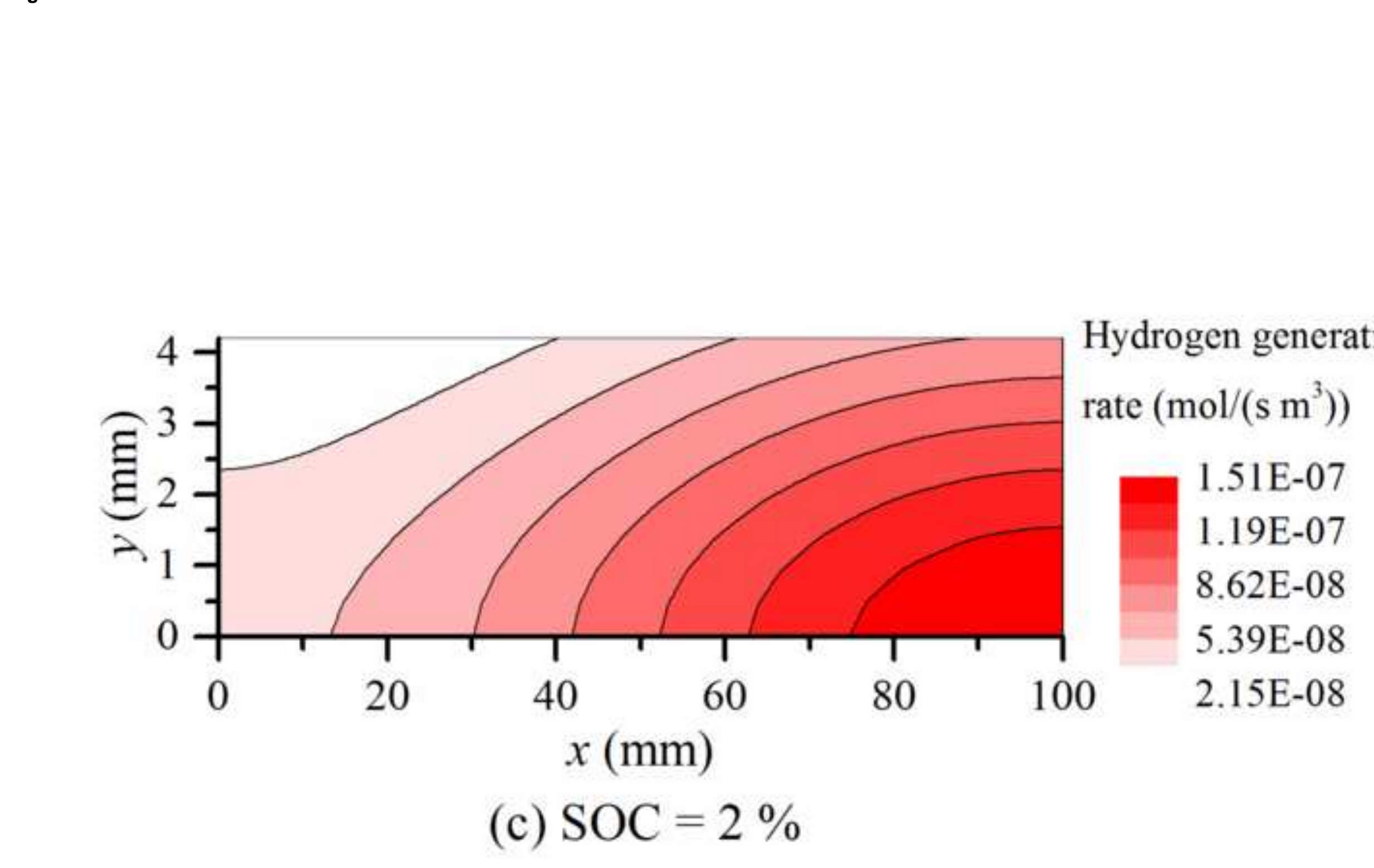

Hydrogen generation

rate $\left(\mathrm{mol} /\left(\mathrm{s} \mathrm{m}^{3}\right)\right)$

1.51E-07

1.19E-07

8.62E-08

$5.39 \mathrm{E}-08$

$$
\begin{array}{cccccc}
0 & 20 \quad \begin{array}{l}
40 \\
x(\mathrm{~mm})
\end{array} \\
& \text { (c) } \mathrm{SOC}=2 \%
\end{array}
$$




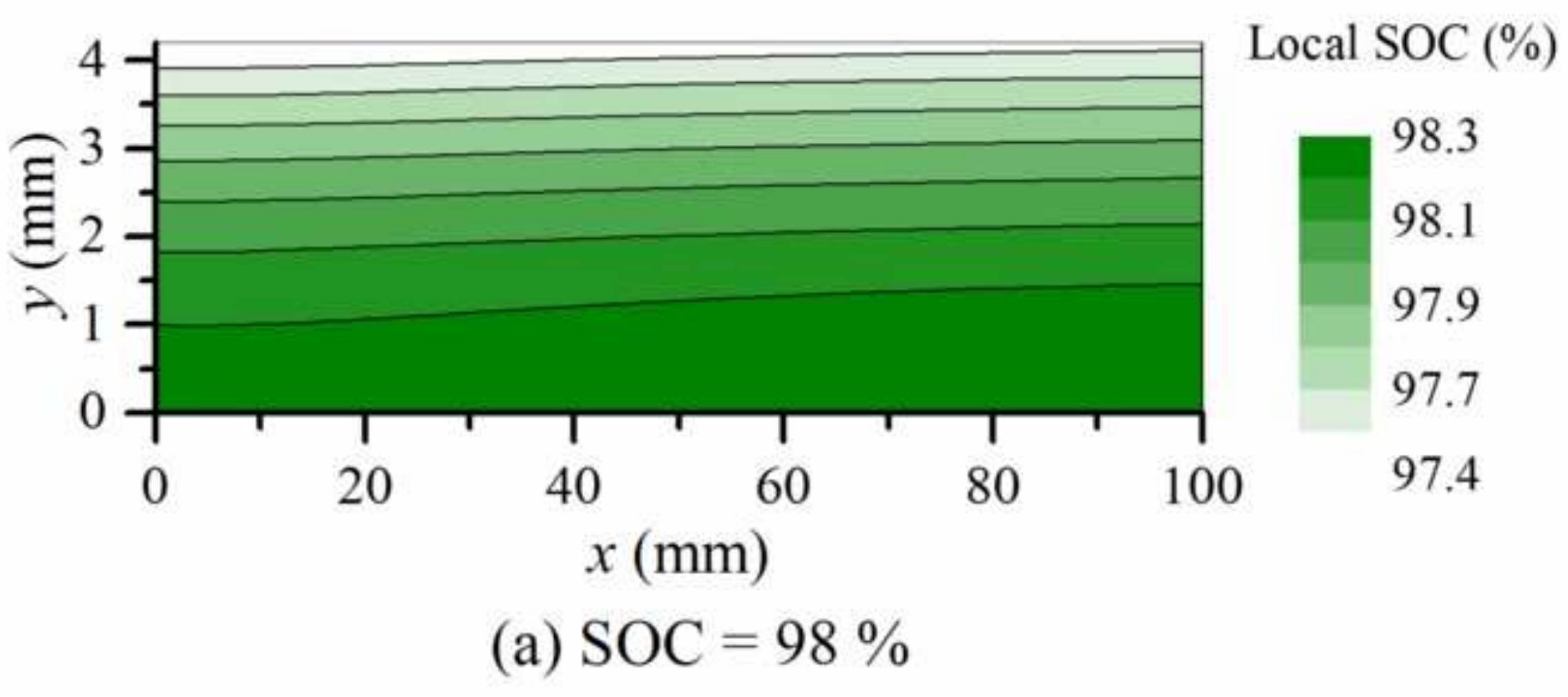

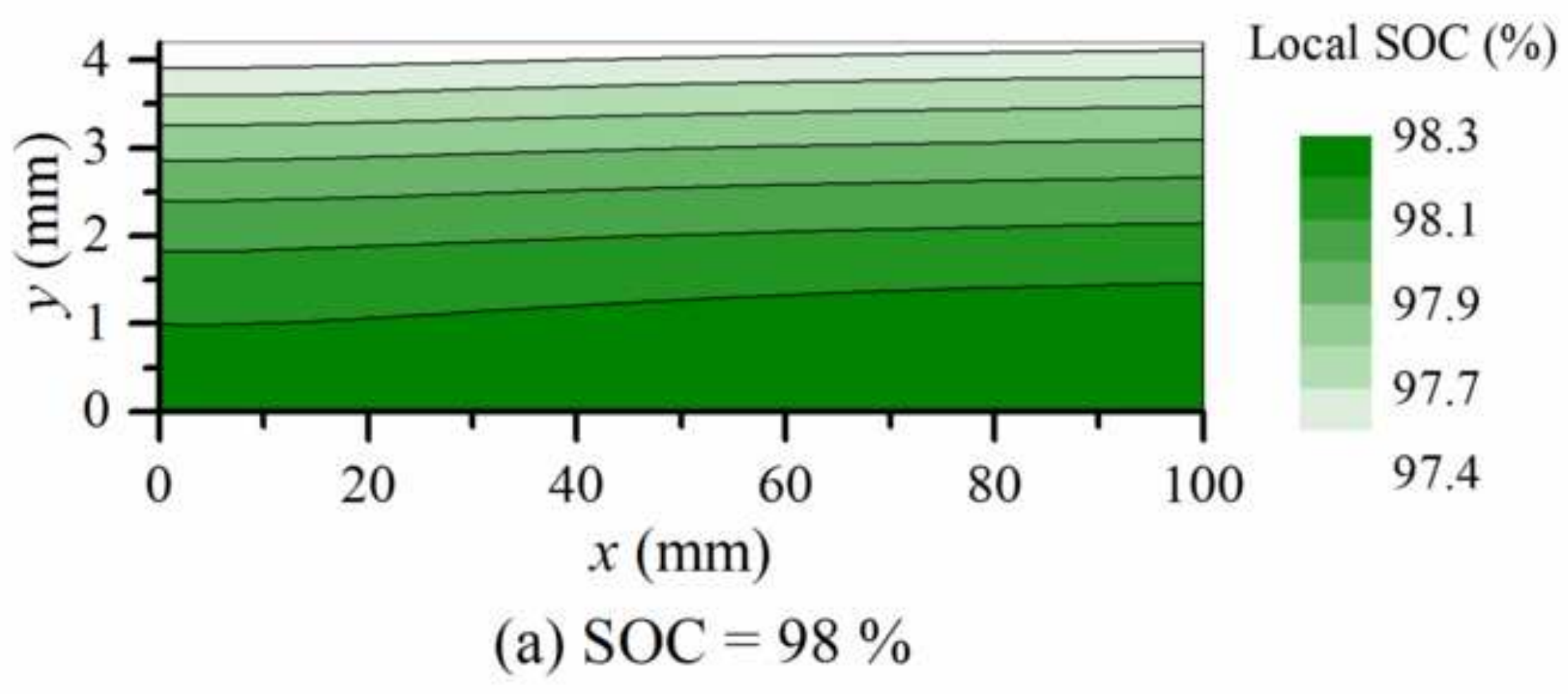

Local SOC (\%)

(a) $\mathrm{SOC}=98 \%$

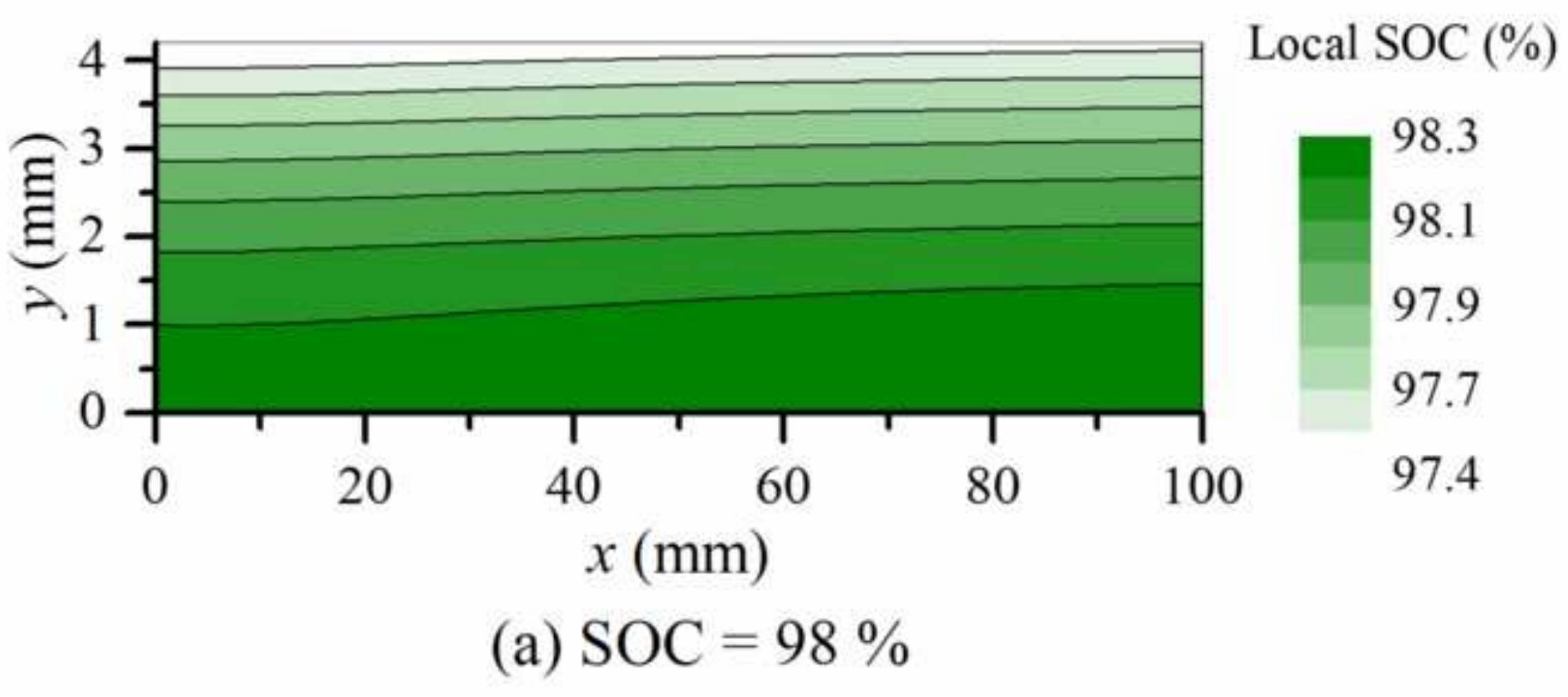

\section{3 \\ 98.1 \\ 97.9 \\ 97.7 \\ 97.4}

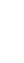

(n)

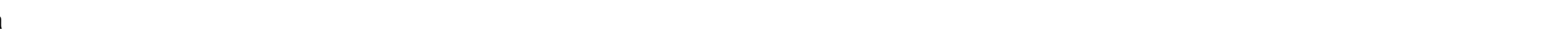




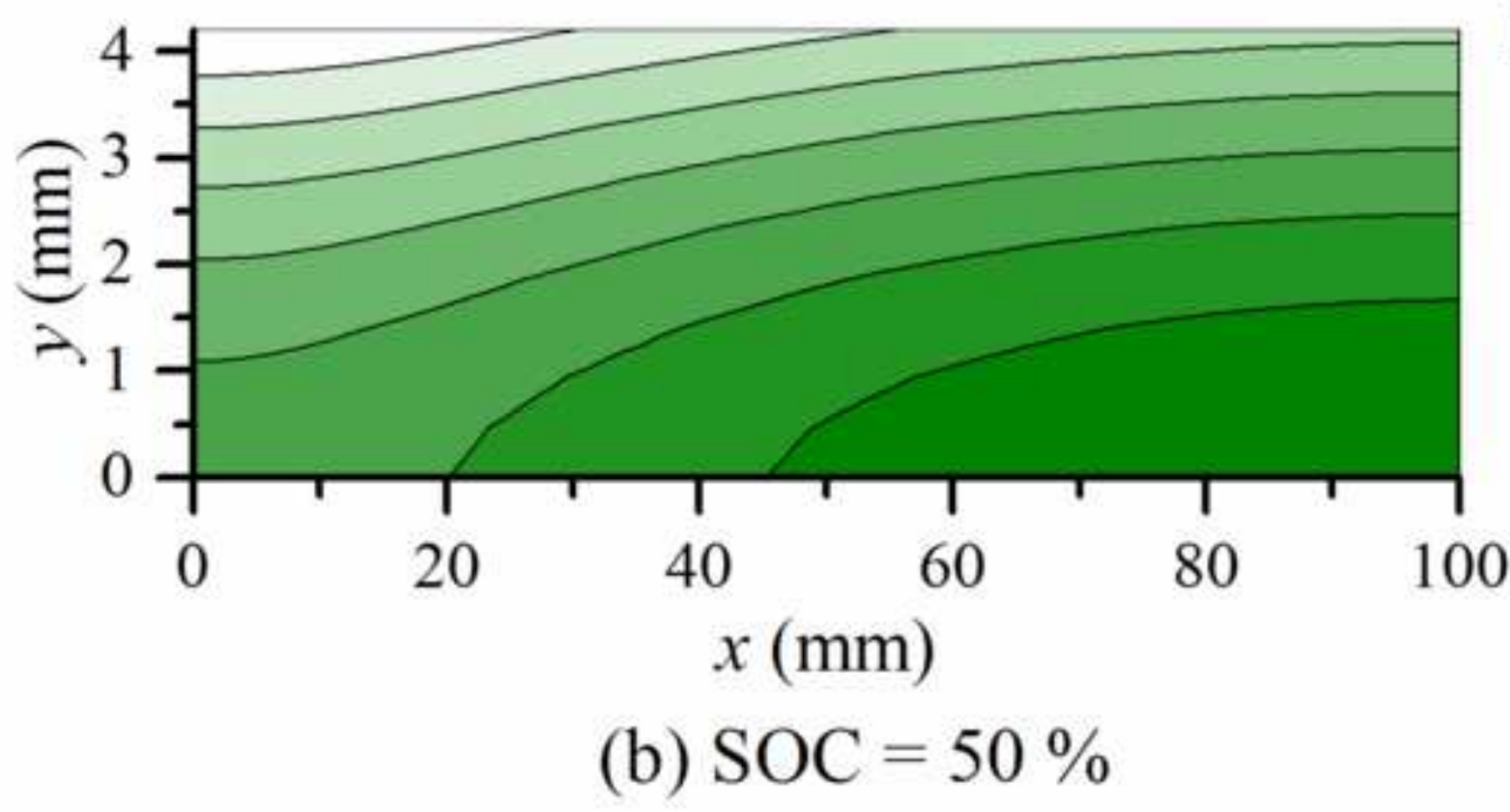

Local SOC (\%)

57.6

52.6

47.5

42.5

37.5

(b) $\mathrm{SOC}=50 \%$

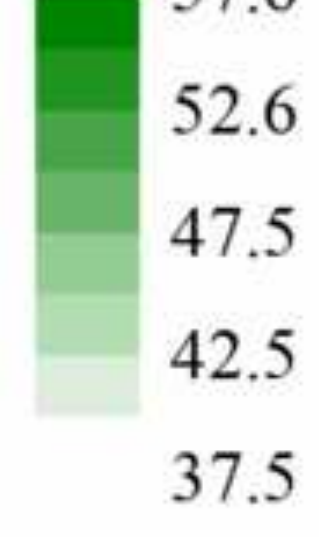

$$
\text { (2) }
$$




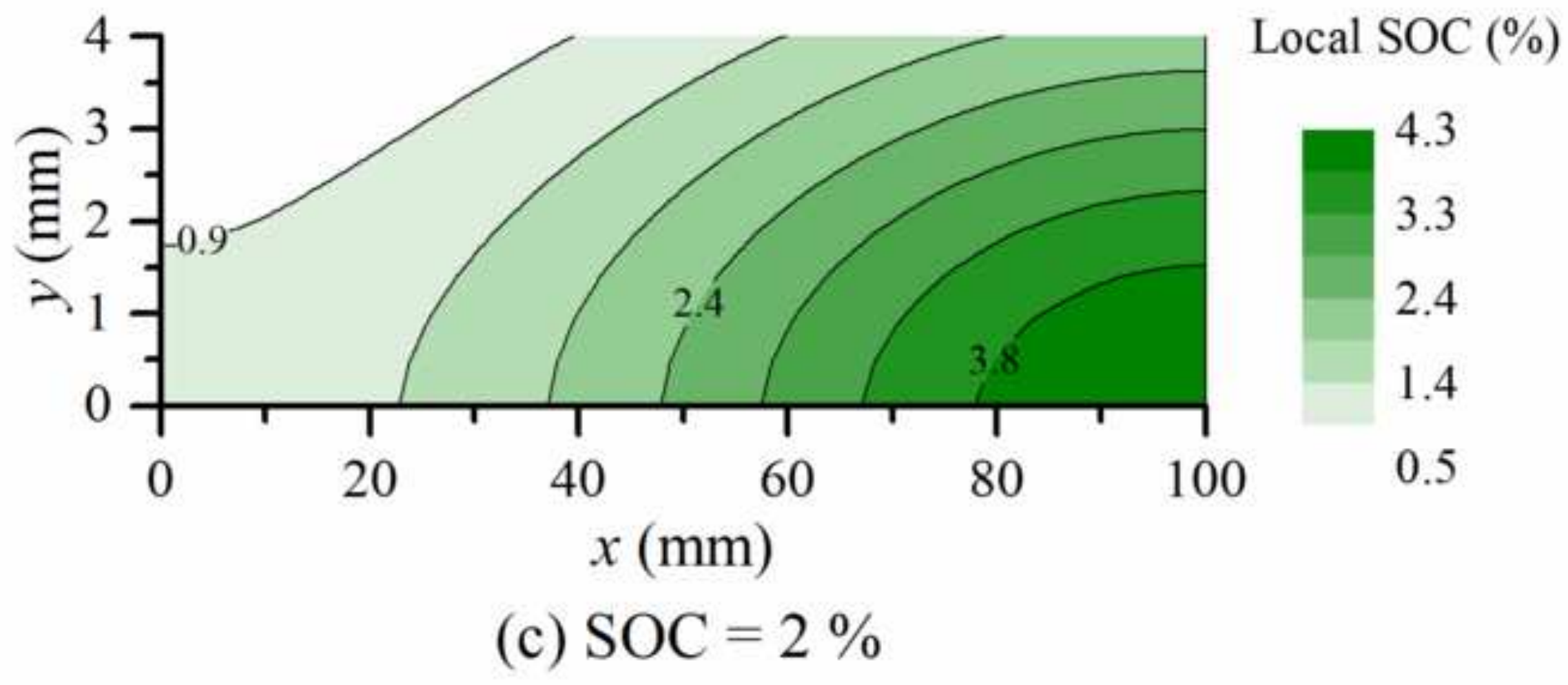

\section{Local SOC (\%)}

4.3

3.3

2.4

1. 4

0.5

(n)




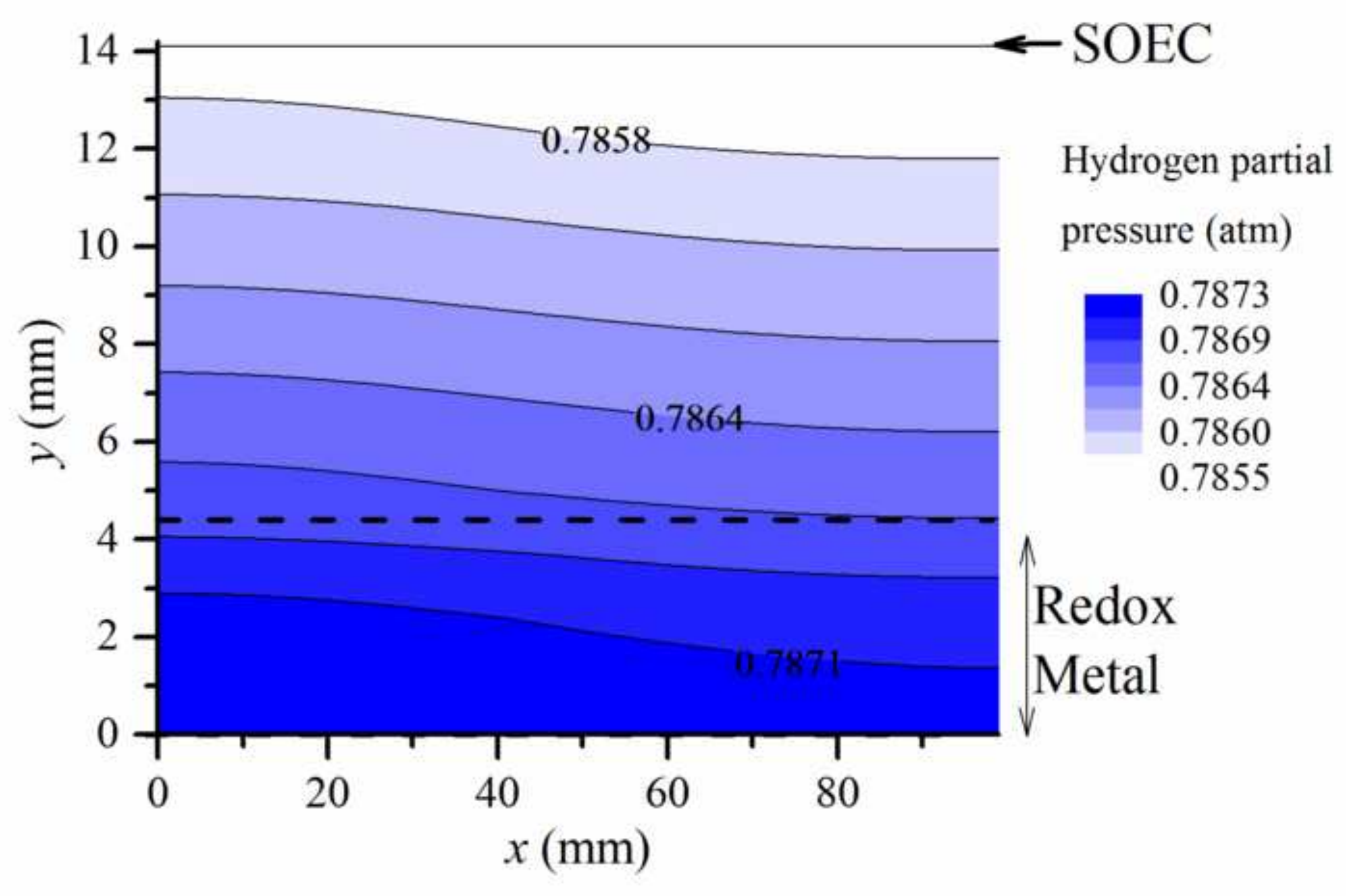

(a) $\mathrm{SOC}=98 \%$

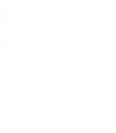

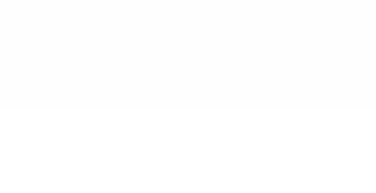

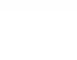

.

(atm)

0.7869
0.7864
0.7860
0.7855
$\times$
1

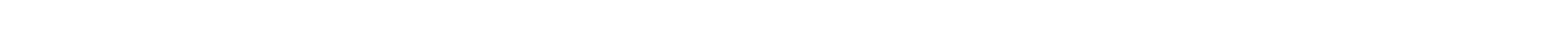




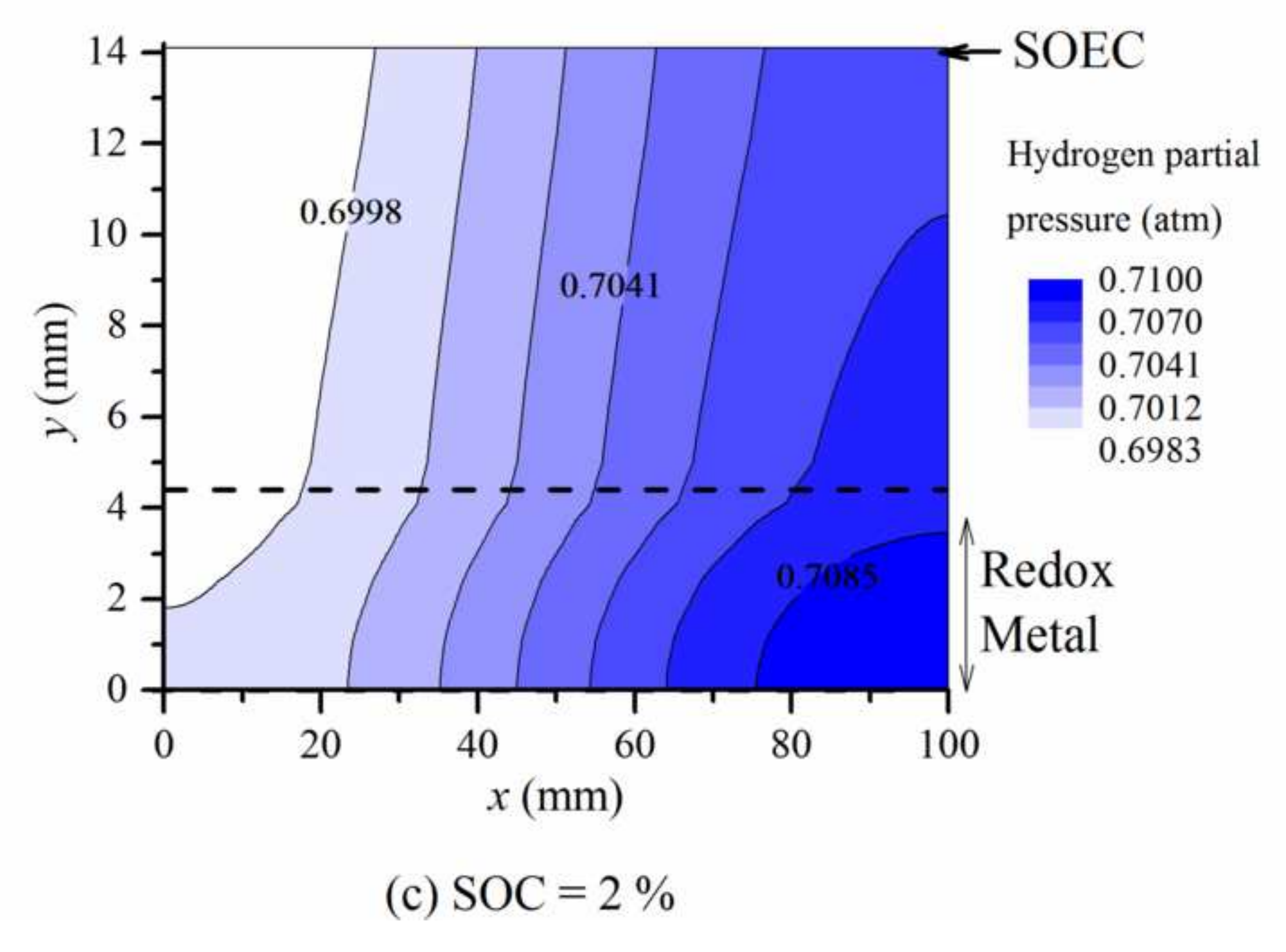

(c) $\mathrm{SOC}=2 \%$

.




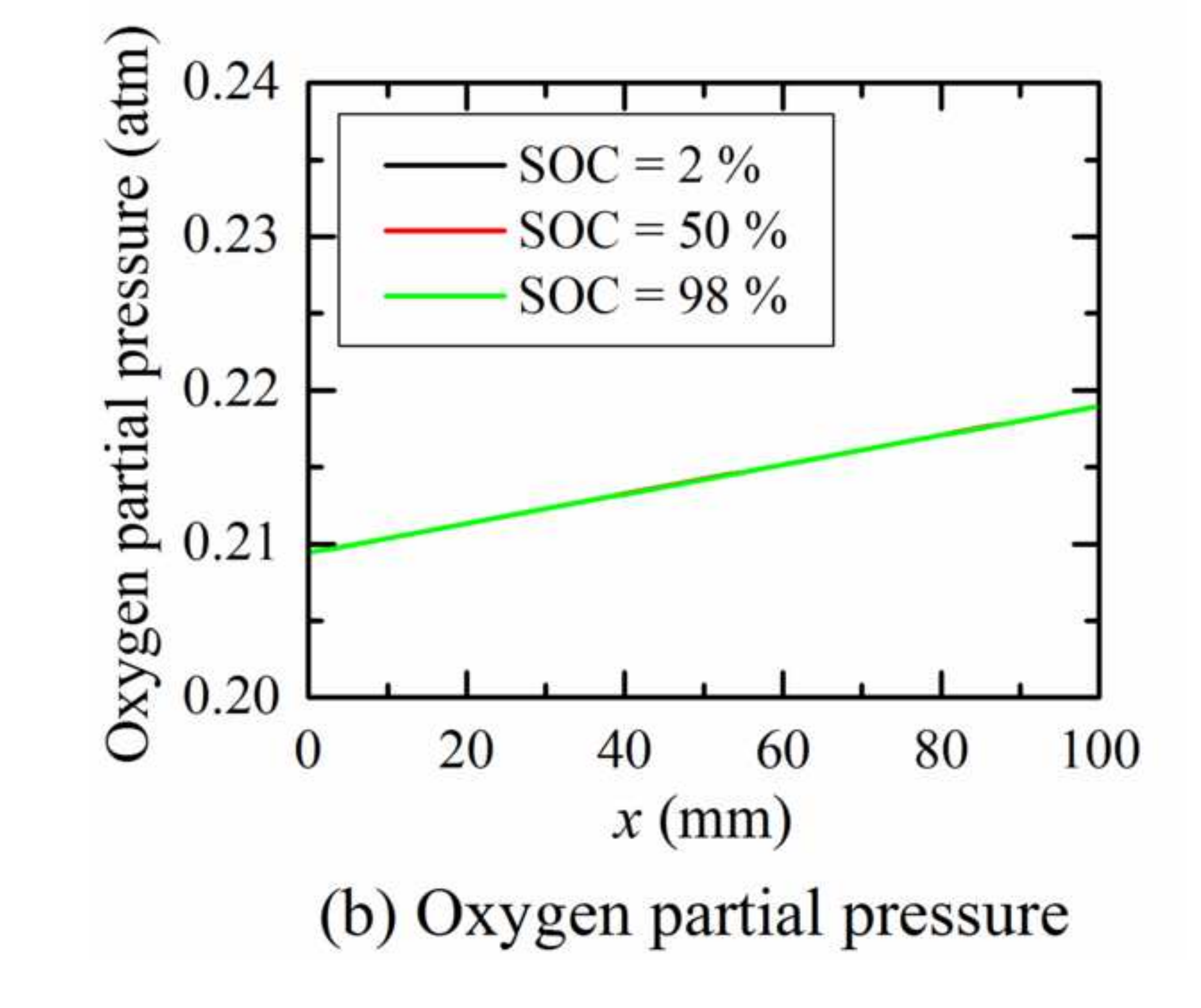

(b) Oxygen partial pressure

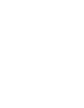

(Fing

(2)

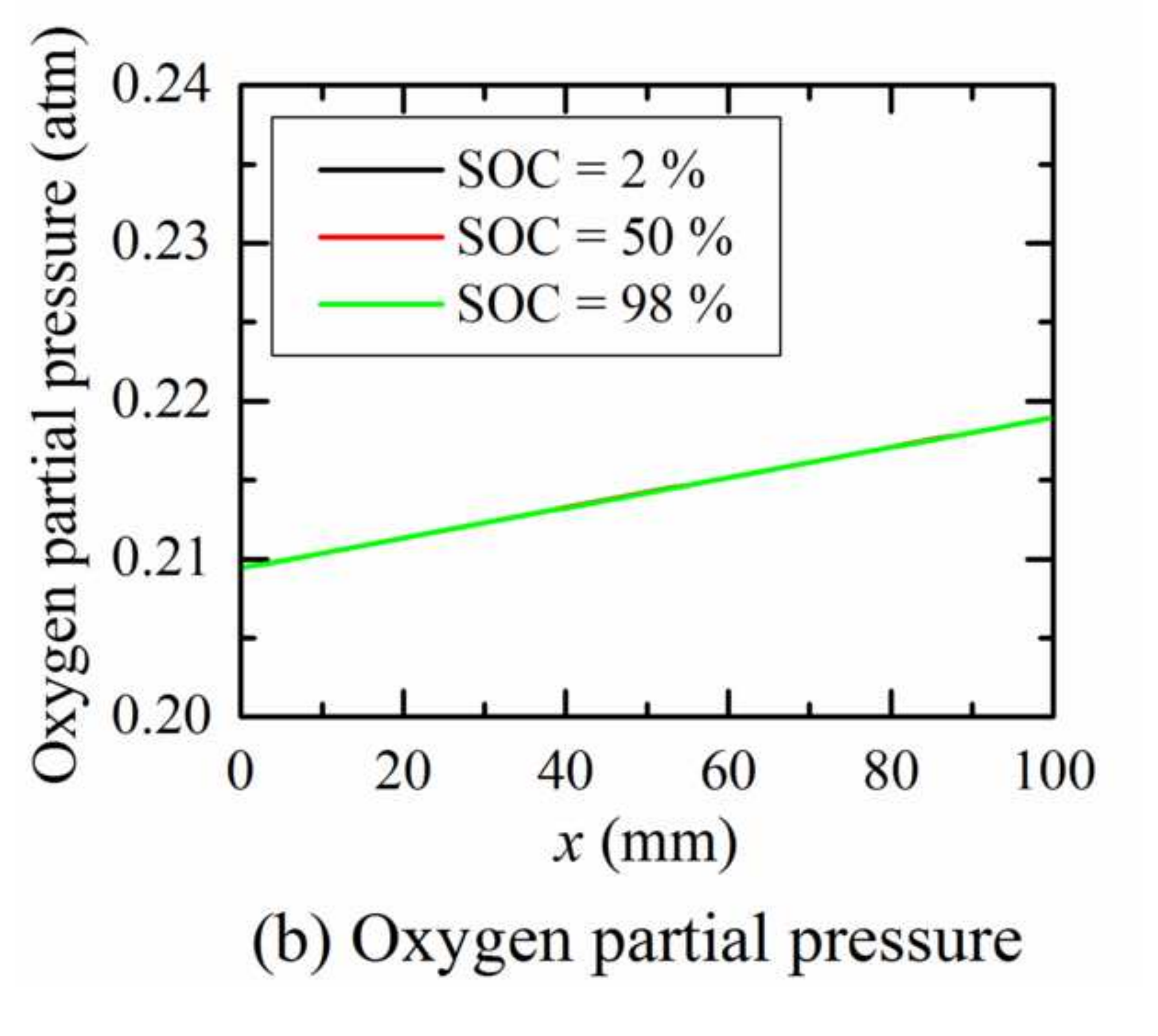

.
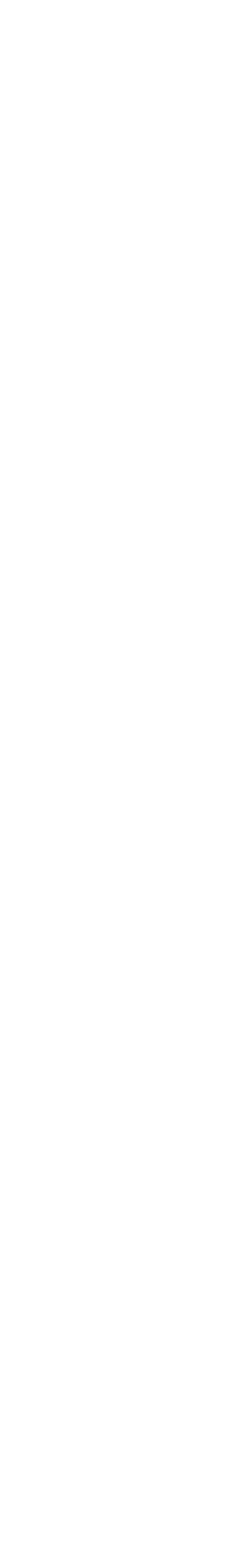

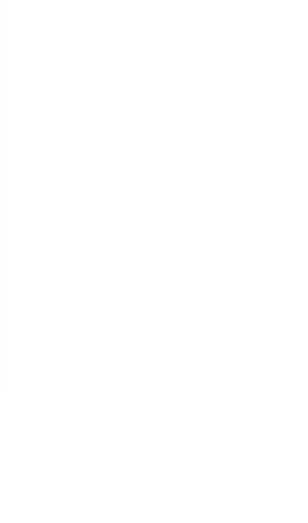




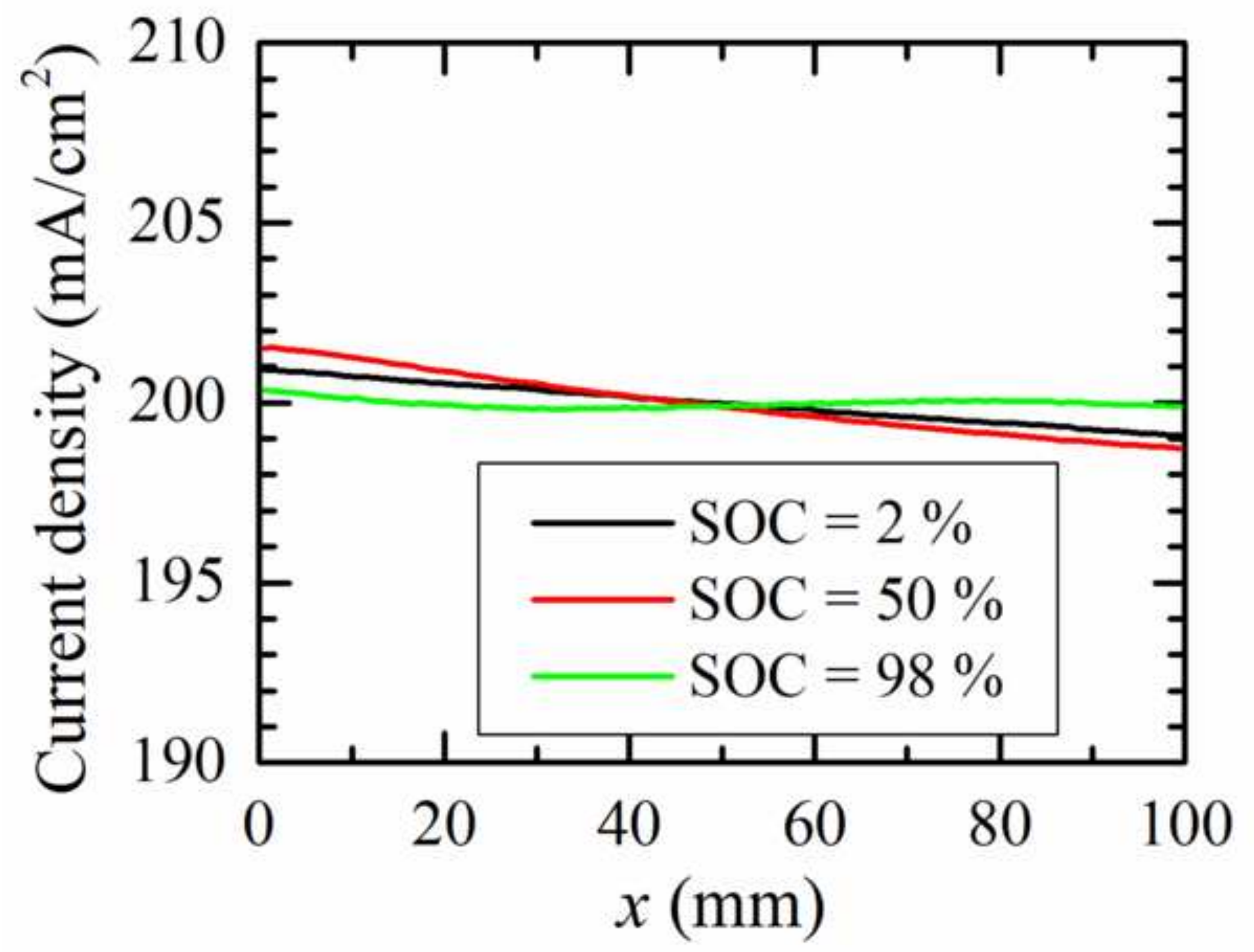

(c) Current density 


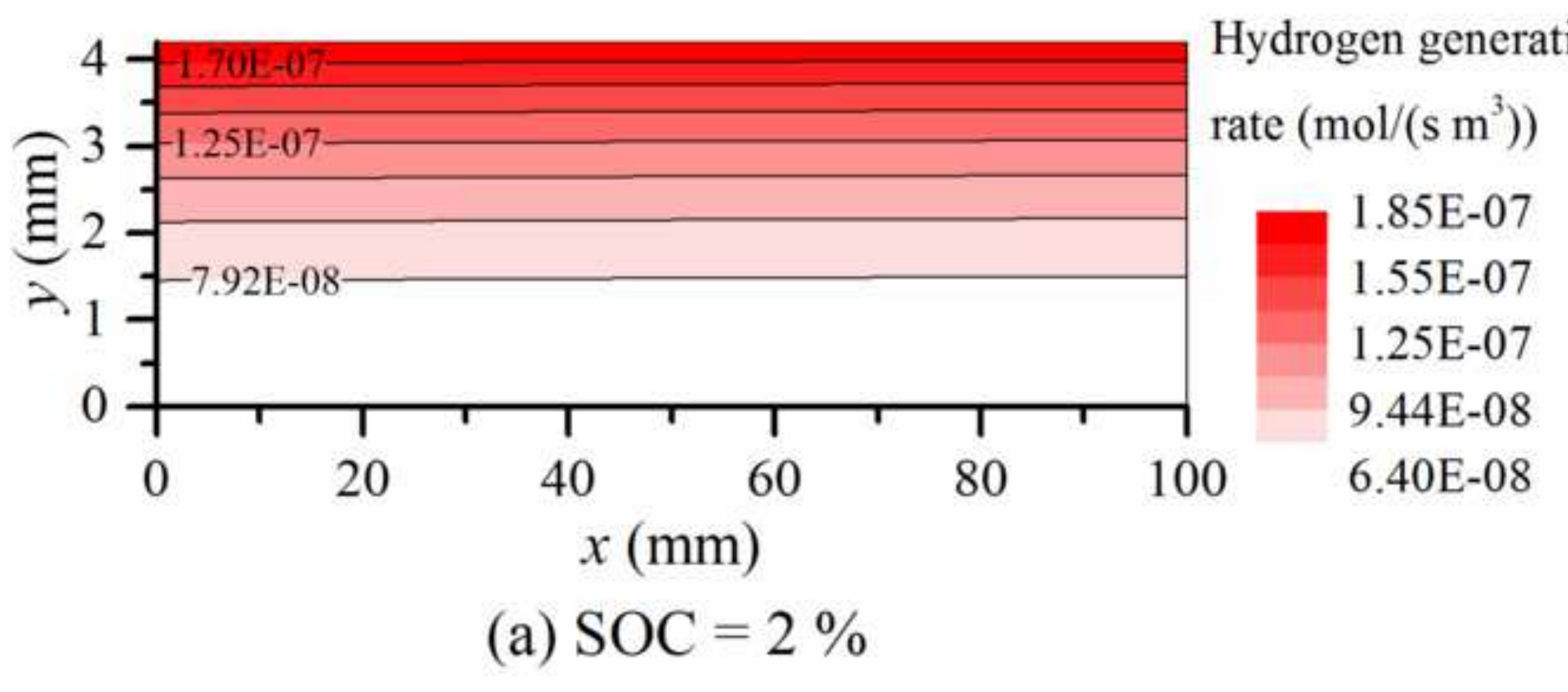

Hydrogen generation rate $\left(\mathrm{mol} /\left(\mathrm{s} \mathrm{m}^{3}\right)\right)$

$1.85 \mathrm{E}-07$

$1.55 \mathrm{E}-07$

$1.25 \mathrm{E}-07$

$9.44 \mathrm{E}-08$ $6.40 \mathrm{E}-08$

(a) $\mathrm{SOC}=2 \%$

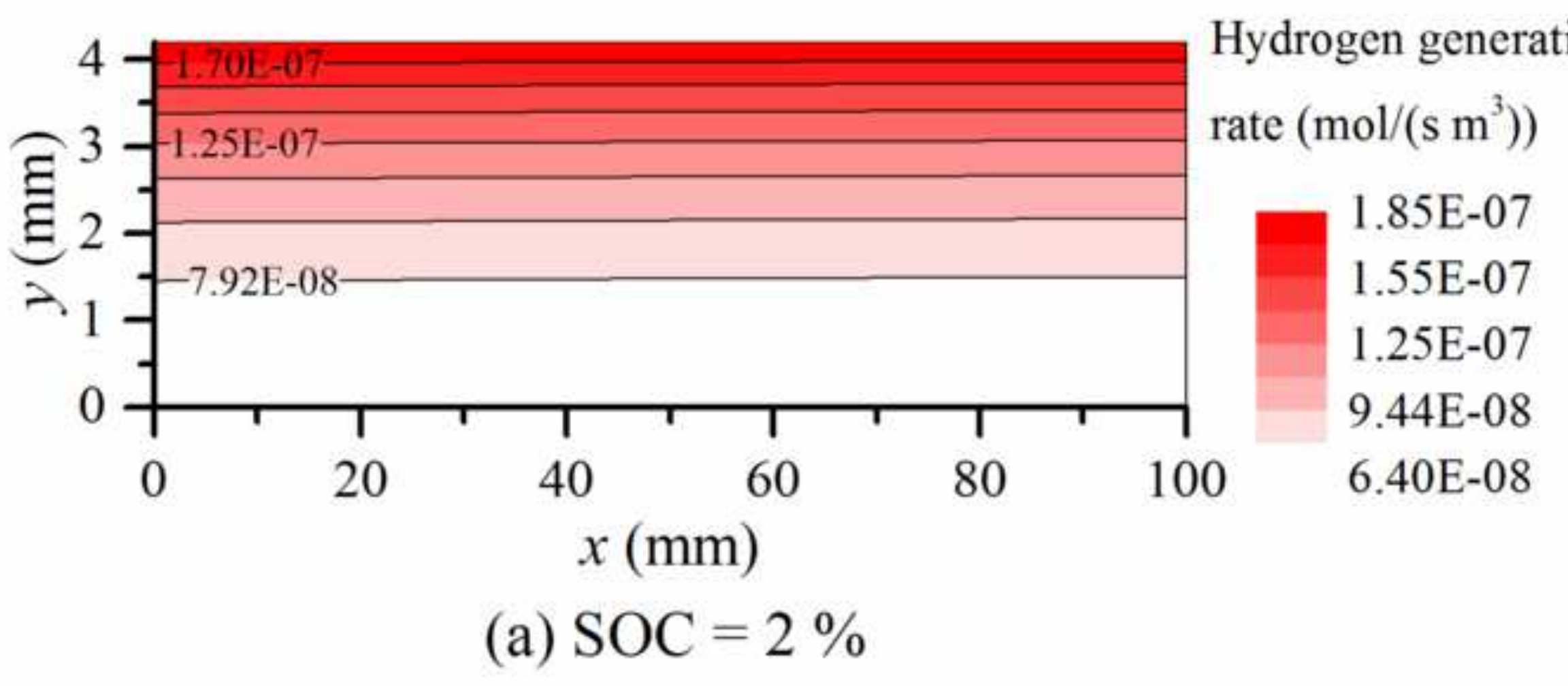




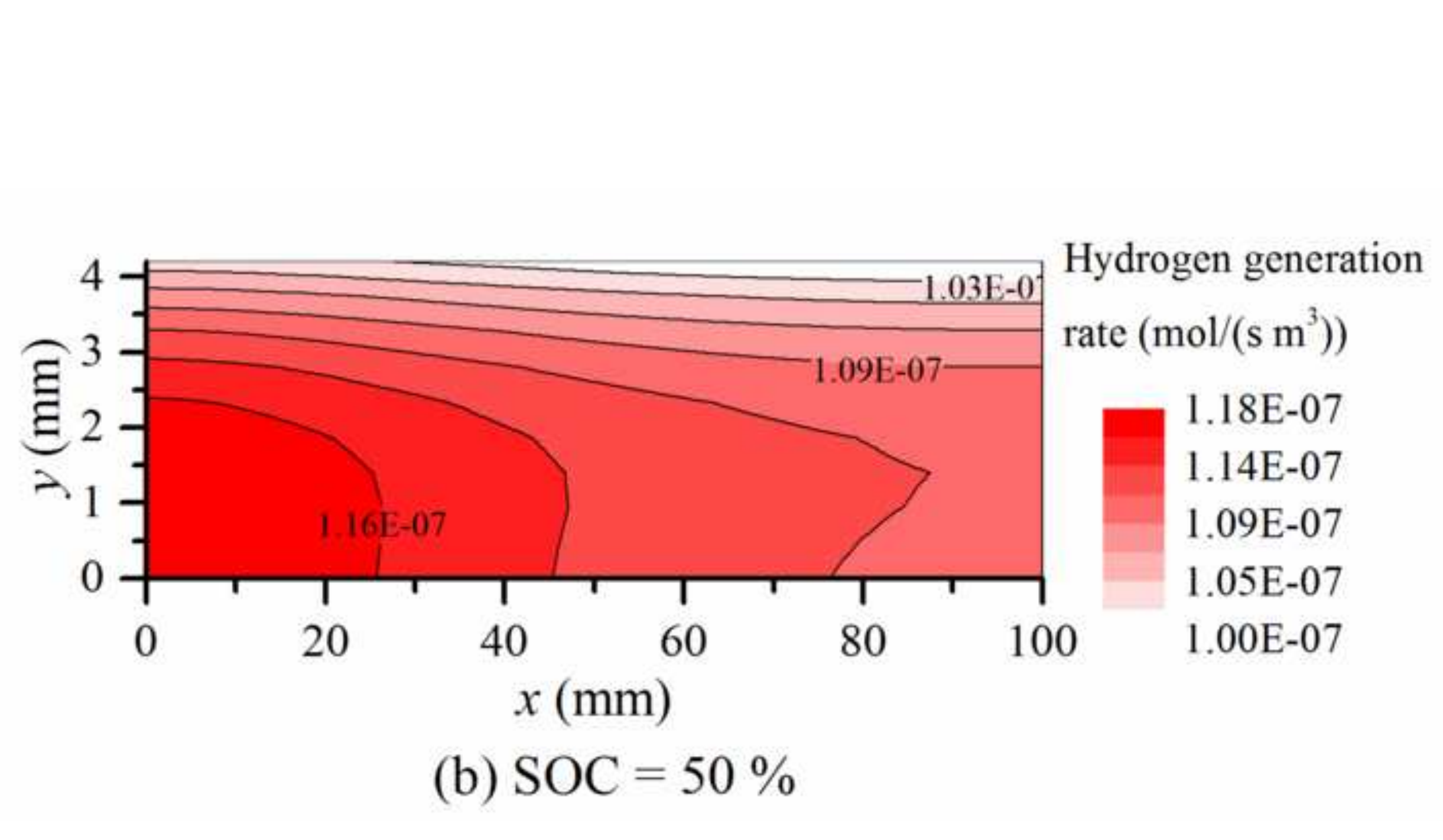

\section{ion}

C

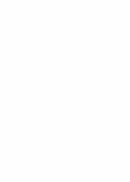

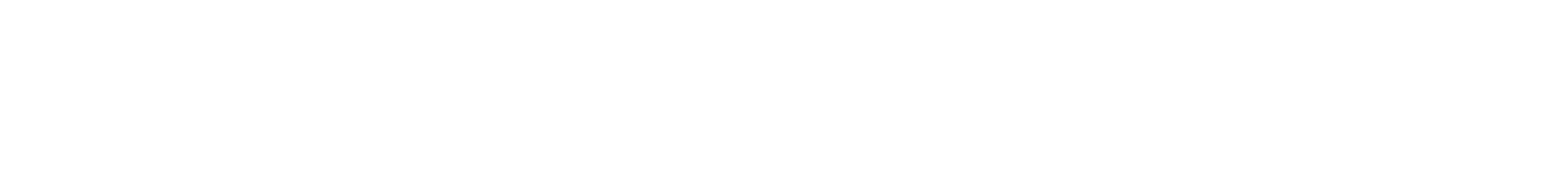

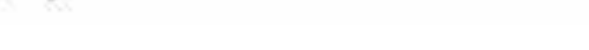




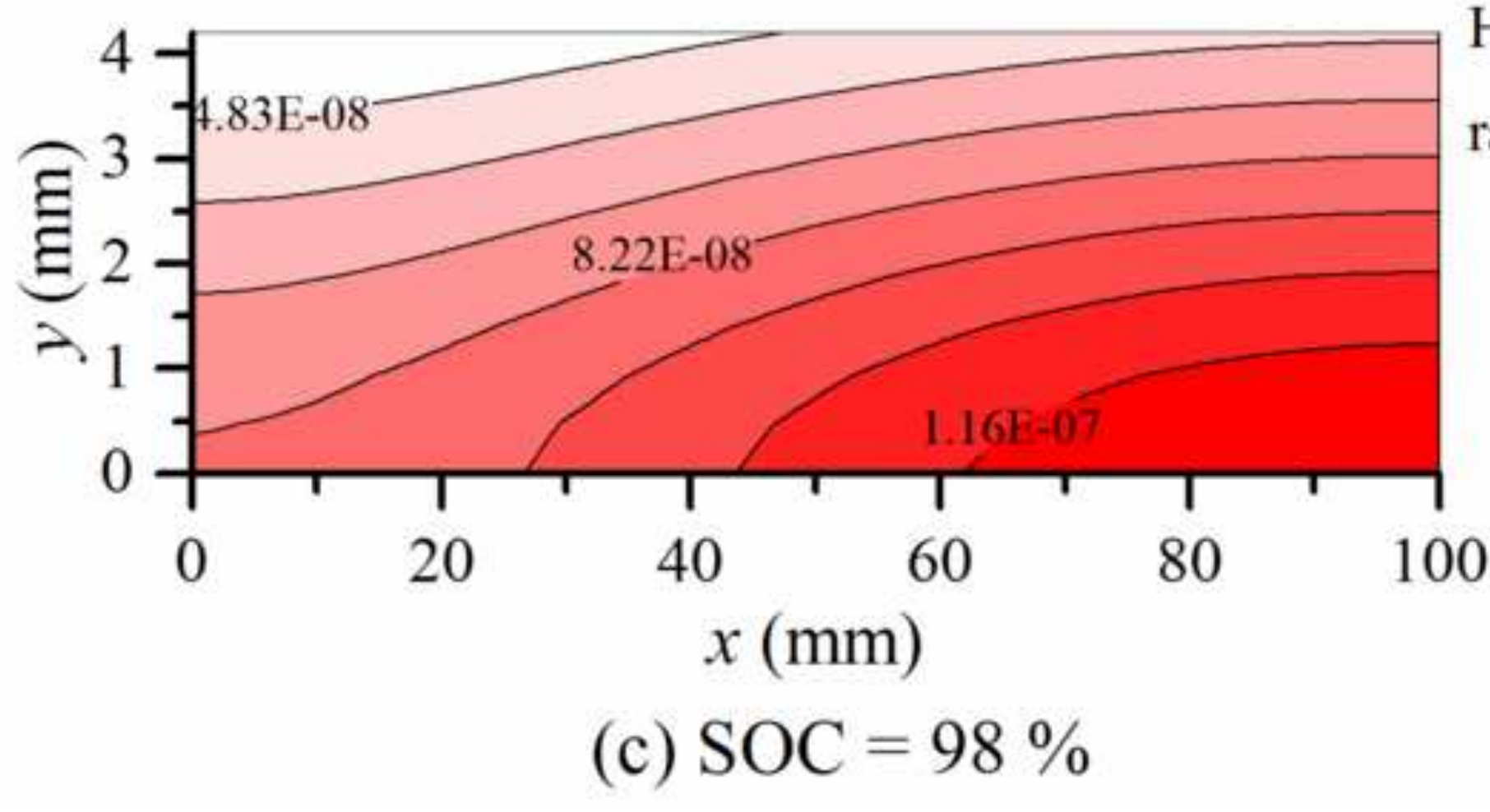

Hydrogen generation rate $\left(\mathrm{mol} /\left(\mathrm{s} \mathrm{m}^{3}\right)\right)$

1.28E-07 $1.05 \mathrm{E}-07$ 8.22E-08 5.96E-08 3.70E-08

(c) $\mathrm{SOC}=98 \%$

$1.28 \mathrm{E}-07$
$1.05 \mathrm{E}-07$
$8.22 \mathrm{E}-08$
$5.96 \mathrm{E}-08$
$3.70 \mathrm{E}-08$

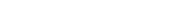




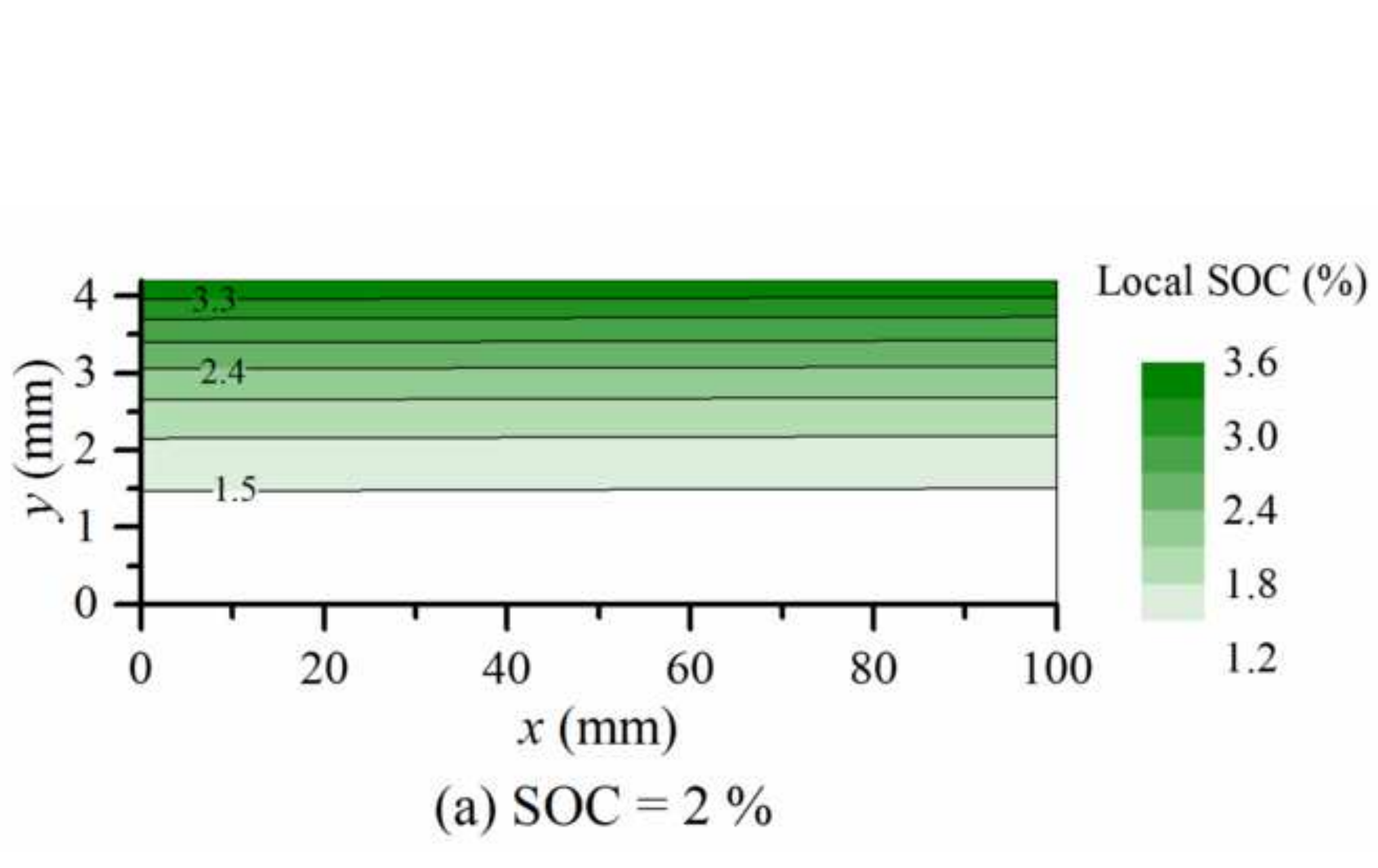




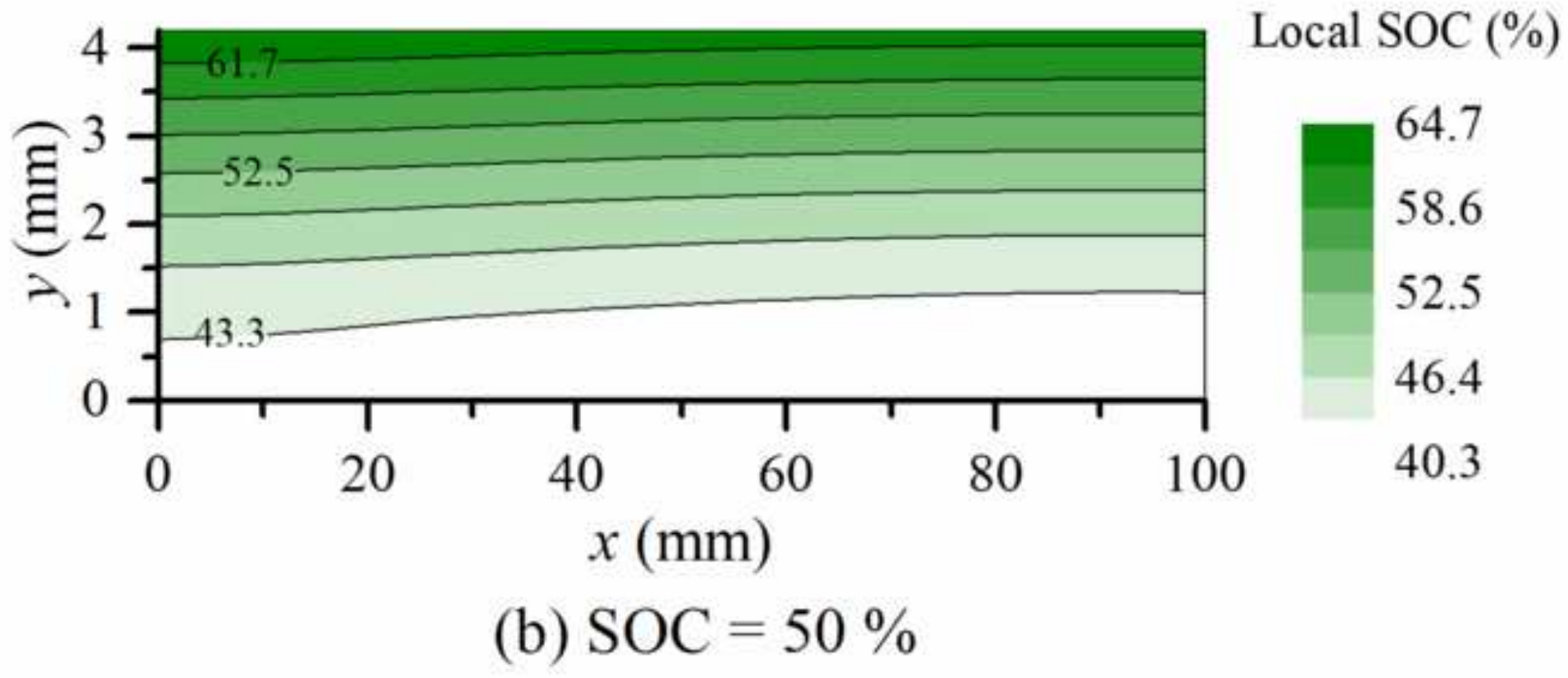

Local SOC (\%)

64.7

58.6

52.5

46.4

40.3

(b) $\mathrm{SOC}=50 \%$ 


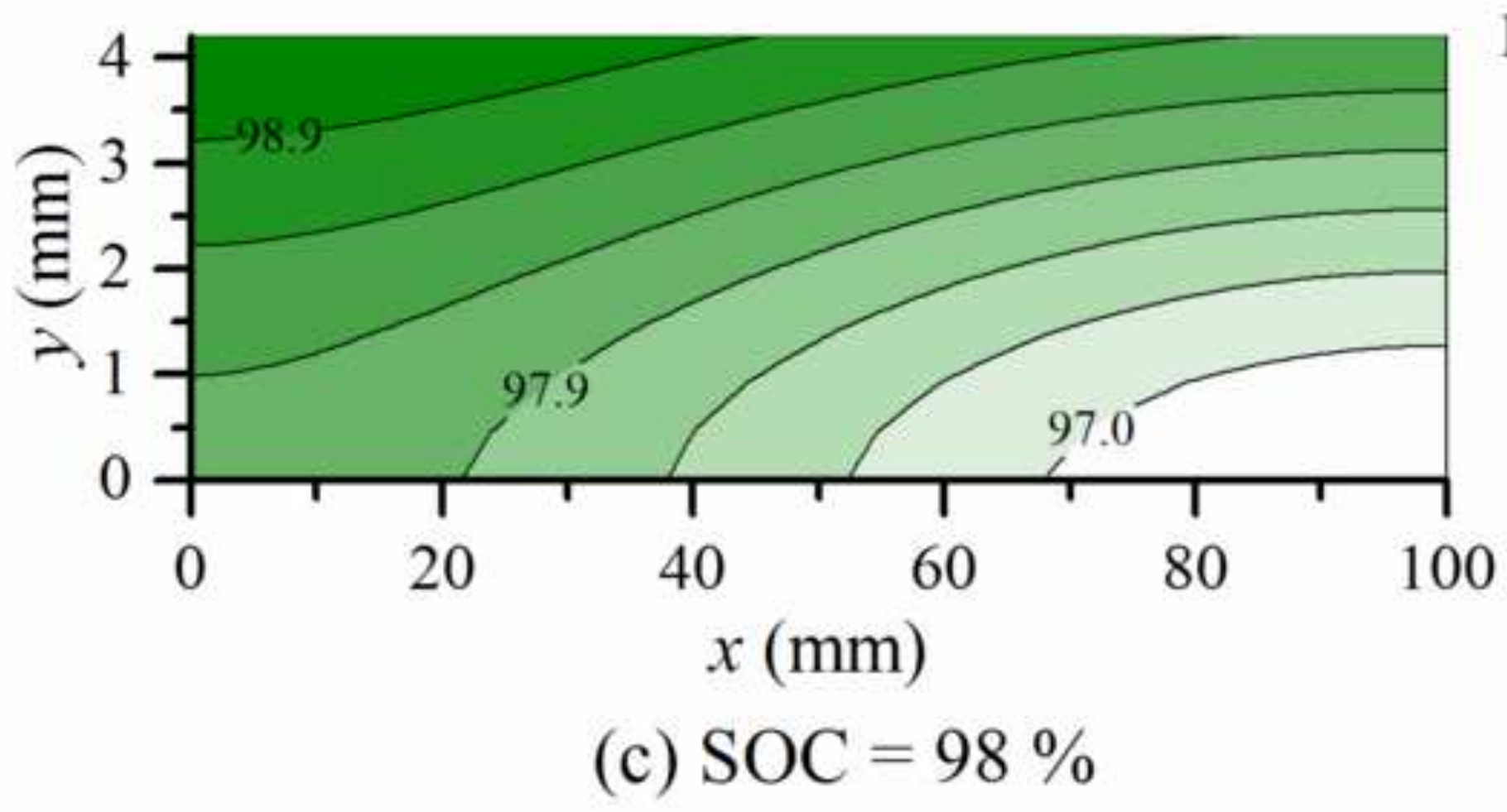

Local SOC (\%)

99.2

98.6

97.9

97.3

96.7

(c) $\mathrm{SOC}=98 \%$
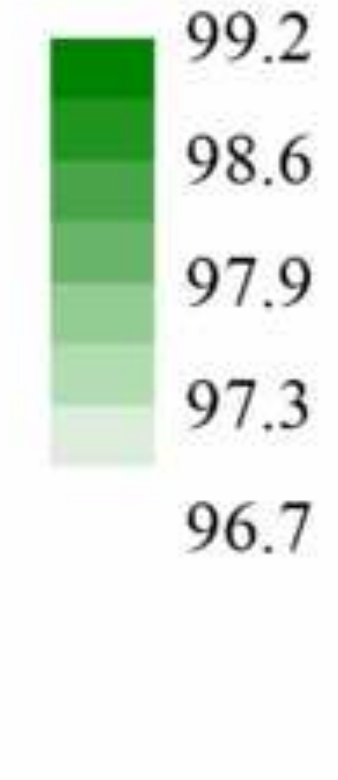

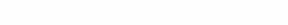




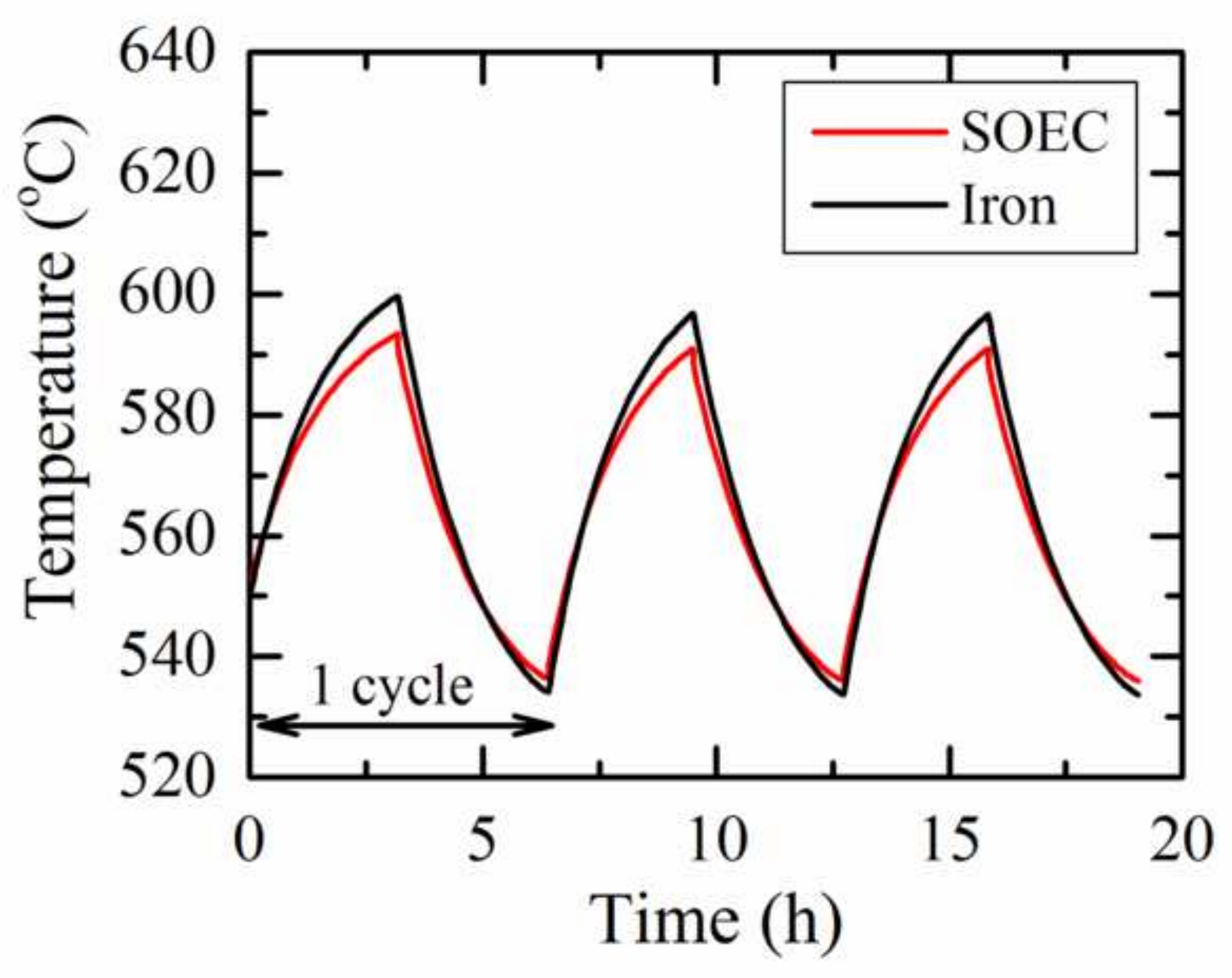

(a) Temperature 


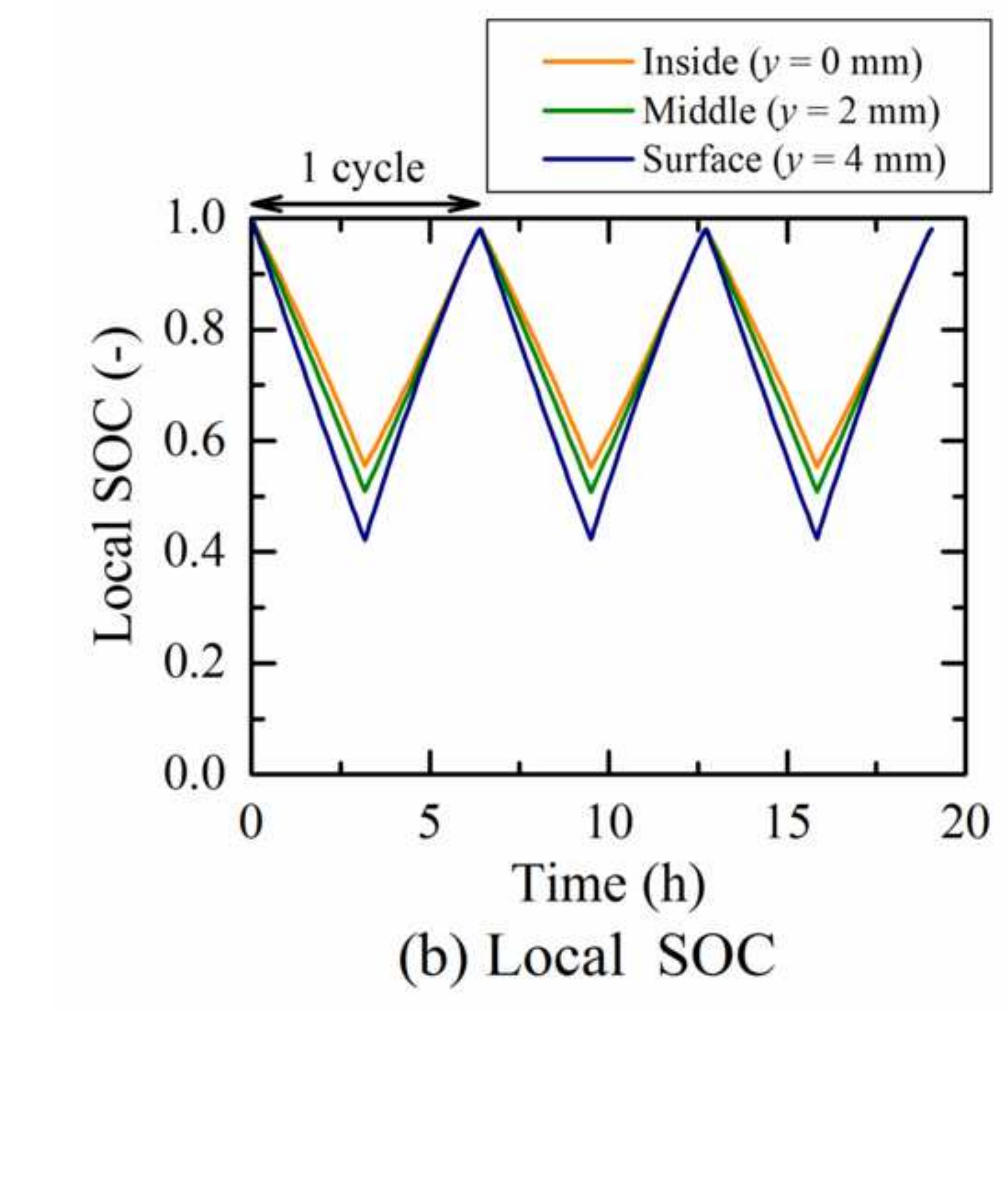

Figure.14b

\footnotetext{
.
}

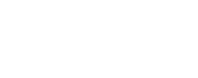

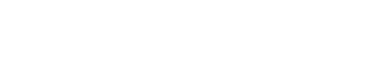
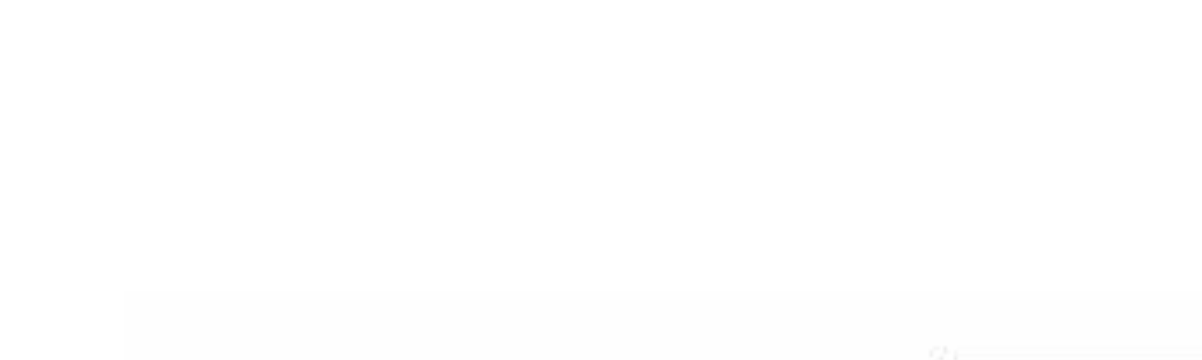


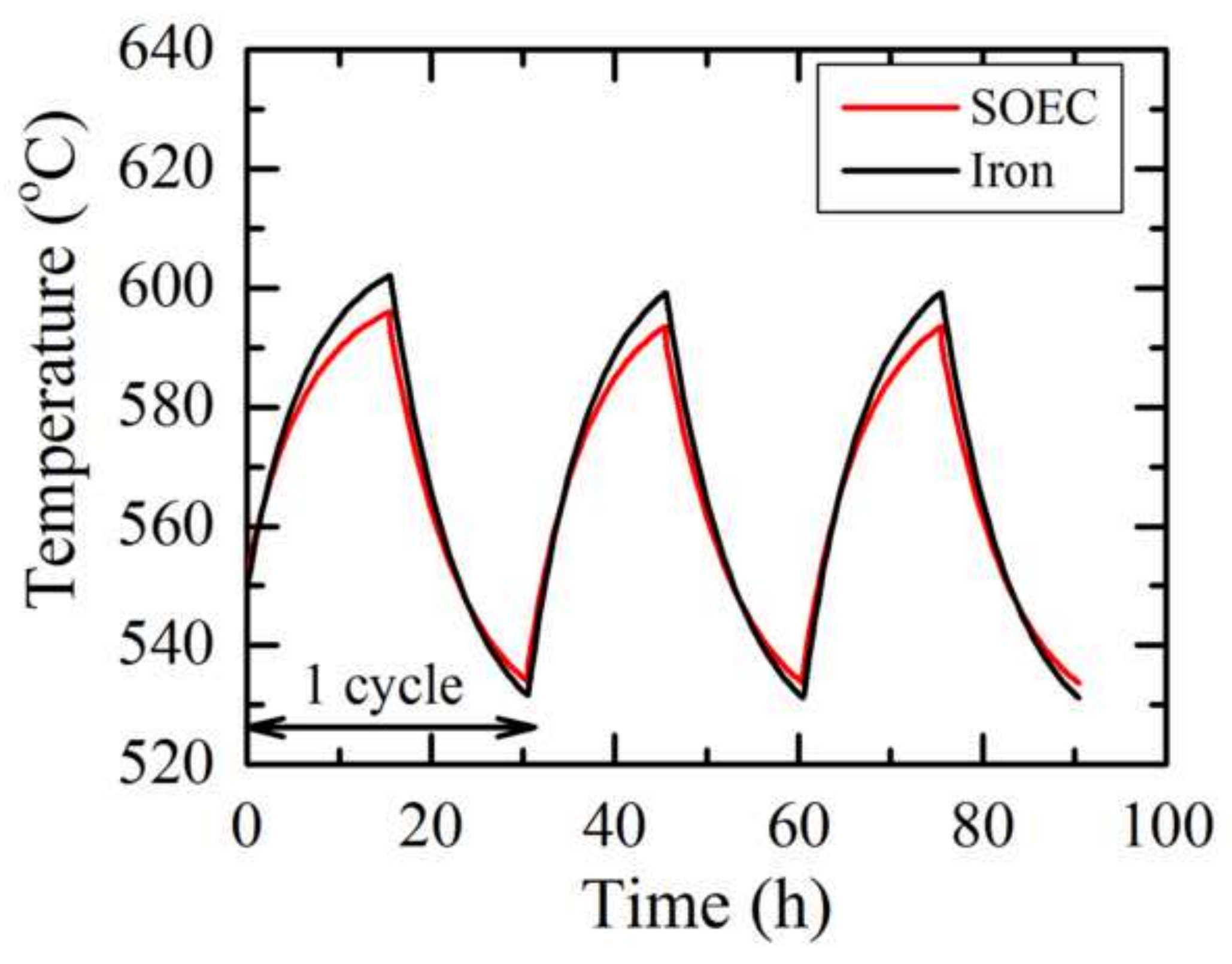

(a) Temperature 


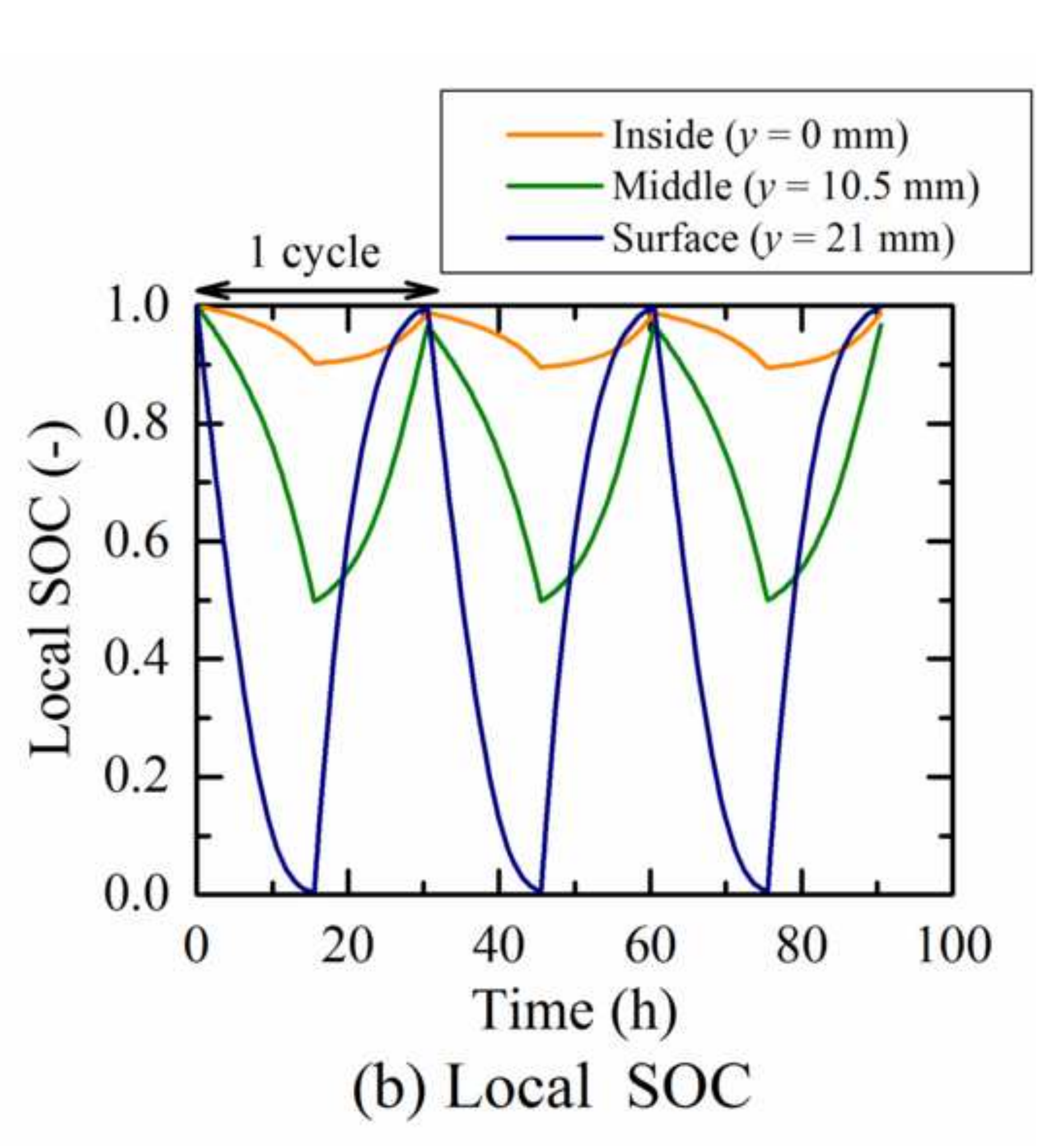

Figure.15b

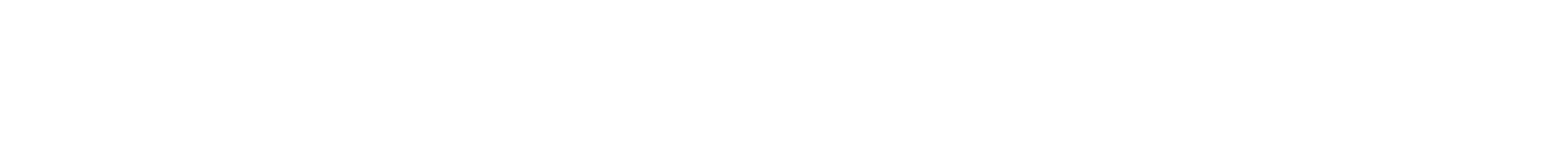
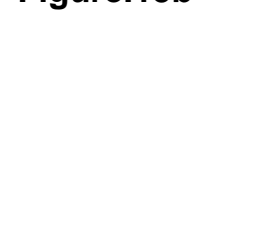